\title{
The effect of macrophage polarization on the expression of the oxytocin signalling system in enteric neurons
}

\author{
Yao Shi ${ }^{1}$, Shuang Li ${ }^{1}$, Haojie Zhang ${ }^{1}$, Jianchun Zhu' ${ }^{1}$, Tongtong Che ${ }^{2}$, Bing Yan ${ }^{3}$, Jingxin Li $i^{1}$ and \\ Chuanyong Liu Li, $^{*}$
}

\begin{abstract}
Background: The aim of the current study was to investigate the effect of macrophage polarization on the expression of oxytocin (OT) and the oxytocin receptor (OTR) in enteric neurons.

Methods: In this study, we used a classic colitis model and D-mannose model to observe the correlation between macrophage polarization and OT signalling system. In order to further demonstrate the effect of macrophages, we examined the expression of OT signalling system after depletion of macrophages.

Results: The data showed that, in vitro, following polarization of macrophages to the M1 type by LPS, the macrophage supernatant contained proinflammatory cytokines (IL-1 $\beta$, IL-6 and TNF- $a$ ) that inhibited the expression of OT and OTR in cultured enteric neurons; following macrophage polarization to the M2 type by IL4, the macrophage supernatant contained anti-inflammatory cytokines (TGF- $\beta$ ) that promoted the expression of OT and OTR in cultured enteric neurons. Furthermore, M1 macrophages decreased the expression of the OT signalling system mainly through STAT3/NF-KB pathways in cultured enteric neurons; M2 macrophages increased the expression of the OT signalling system mainly through activation of Smad2/3 and inhibition of the expression of Peg 3 in cultured enteric neurons. In a colitis model, we demonstrated that macrophages were polarized to the M1 type during the inflammatory phase, with significant decreased in the expression of OT and OTR. When macrophages were polarized to the M2 type during the recovery phase, OT and OTR expression increased significantly. In addition, we found that D-mannose increased the expression of OT and OTR through polarization of macrophages to the M2 type.
\end{abstract}

Conclusions: This is the first study to demonstrate that macrophage polarization differentially regulates the expression of OT and OTR in enteric neurons.

Keywords: OT signalling system, Macrophage polarization, Proinflammatory and anti-inflammatory factors, Neuralimmune interactions

\footnotetext{
*Correspondence: liucy@sdu.edu.cn

1 Department of Physiology, School of Basic Medical Sciences, Cheeloo

Medical College, Shandong University, 44 Wenhua Xi Road, Jinan 250012, Shandong, People's Republic of China

Full list of author information is available at the end of the article
}

\section{Introduction}

Oxytocin (OT) is traditionally considered a nonapeptide hormone synthesized in the hypothalamus that is released from the posterior pituitary into circulation $[1,2]$ and is involved in milk let-down and uterine contraction [3]. Recent studies have shown that the oxytocin receptor (OTR) is widely present in the central and peripheral systems [3-5]. The role of OT is very broad; original author(s) and the source, provide a link to the Creative Commons licence, and indicate if changes were made. The images or other third party material in this article are included in the article's Creative Commons licence, unless indicated otherwise in a credit line to the material. If material is not included in the article's Creative Commons licence and your intended use is not permitted by statutory regulation or exceeds the permitted use, you will need to obtain permission directly from the copyright holder. To view a copy of this licence, visit http://creativecommons.org/licenses/by/4.0/. The Creative Commons Public Domain Dedication waiver (http://creativeco mmons.org/publicdomain/zero/1.0/) applies to the data made available in this article, unless otherwise stated in a credit line to the data. 
it not only participates in the regulation of certain social behaviours [6] but also relieves colitis through preventing the free radical damage cascade [7]. OT also promotes the proliferation and differentiation of adipose tissuederived mesenchymal stem cells into nerve cells [8]. Studies in our laboratory and others have made clear that OT is an endogenous neuropeptide in the gastrointestinal tract and regulates the secretion, motility, and immunity of the digestive tract [9-11]. OT is mainly expressed in the myenteric nerve plexus and submucosal nerve plexus, including neuron bodies and nerve fibres [12].

The intestine is the largest immune organ in the body and contains various innate immune and adaptive immune cells. Macrophages are the most important innate immune cells [13] and are mainly distributed in the lamina propria and muscular layer. Lamina propria macrophages (LMs) are involved in the regulation of intestinal inflammation and have been identified as the main innate immune cells that induce inflammatory bowel disease (IBD) [14, 15]. Muscularis macrophages (MMs) are densely distributed and adjacent to enteric neurons and nerve fibres. There is a direct synaptic connection between macrophages and neurons [16]. This connection facilitates the dialogue between the enteric nervous system (ENS) and macrophages through neurotransmitters, growth factors, cytokines and hormones [17-19]. Tracey et al. proposed that the excitatory neurotransmitter acetylcholine targets resident macrophages to inhibit the production of inflammatory mediators [20]. Tissue-resident MMs protect enteric-associated neurons through the $\beta 2$-adrenergic receptor and arginase 1 polyamine axis after infection [21].

We previously demonstrated that OT regulates the polarization of macrophages to relieve intestinal inflammation [22]. OTR-deficient mice are more susceptible to 2,4,6-trinitrobenzenesulfonic acid (TNBS)- and dextran sulfate sodium (DSS)-associated colitis [12]. Local and systemic levels of intestinal OT change during intestinal inflammation $[9,10]$. Therefore, we assumed that inflammatory factors released by macrophages might regulate the expression of OT and OTR. This article aimed to explore whether the polarization of macrophages regulates the expression of the OT signalling system in the ENS, which might be of great significance for maintaining intestinal homeostasis.

\section{Materials and methods Reagents}

Lipopolysaccharide was purchased from Sigma-Aldrich (St Louis, Ca). Recombinant mouse IL-4, recombinant mouse IL-6, recombinant mouse IL- $1 \beta$, recombinant mouse TNF- $\alpha$, recombinant mouse IL-10, and recombinant mouse TGF- $\beta$ were purchased from R\&D Systems
(Minneapolis, MN). Tocilizumab, LY2109761, R-7050, Stattic, Bay 11-7082, and SIS3HCl were purchased from Selleck (Huston, USA). IL-1 receptor antagonist (IL1Ra) was purchased from Make Research Easy (Nanjing, China). Monoclonal mouse anti- $\beta$-actin (TA-09) was purchased from Zhongshan Golden Bridge Biotechnology (Beijing, China). Antibodies against OT, OTR, $\beta$-tubulin, CD206, F4-80, phospho-STAT3, STAT3, phospho-SMAD2, SMAD2, phospho-SMAD3, SMAD3, p65, and phospho-p65 were purchased from Abcam (Cambridge, UK) and Cell Signaling Technology (Danvers, MA). Secondary antibodies were purchased from Invitrogen Life Technology (Foster City, CA). Mouse ELISA kits were obtained from R\&D Systems and CUSABIO (Wuhan, China). All reagents were analytical grade.

\section{Cell isolation and culture}

The RAW264.7 macrophage-like cell line was purchased from the Cell Bank of the Chinese Academy of Science (Shanghai, China). Cells were cultured in Dulbecco's modified Eagle's medium (HyClone, Logan, Utah) supplemented with $10 \%$ heat-inactivated foetal bovine serum (Gibco, Foster City, CA) and 1\% penicillin-streptomycin solution (Gibco) in a humidified incubator with $5 \% \mathrm{CO}_{2}$ at $37^{\circ} \mathrm{C}$.

Enteric neurons were obtained from C57BL6/J mice as previously described [23]. Briefly, the mice were euthanized with no pain, and the outer skin of the peritoneum was cut. 3-5 cm of colon tissue was excised and immediately placed in a Sylgard ${ }^{\mathrm{TM}}$-lined dissecting dish containing carbogen-gassed Krebs saline. The composition of the Krebs saline was as follows (in $\mathrm{mM}$ ): $\mathrm{NaCl} 120.6$, $\mathrm{KCl} 5.9, \mathrm{CaCl}_{2} 2.5, \mathrm{KH}_{2} \mathrm{PO}_{4} 1.2, \mathrm{MgCl}_{2} 1.2$, and $\mathrm{NaHCO}_{3}$ 15.4. The mesentery was cut with Venus scissors, and the colonic segment was cut longitudinally along the mesentery. The colonic segment was fixed in a dissecting dish, and the contents were thoroughly washed. The mucosa, submucosa, serosal layer, and circular muscle layers were carefully removed with Venus forceps to expose the longitudinal muscle myenteric plexus (LMMP). The LMMP was incubated in Krebs saline containing papain $(10 \mathrm{mg} /$ ml) (P8150, Solarbio, Beijing, China) at $37^{\circ} \mathrm{C}$ for $50 \mathrm{~min}$. The LMMP was stretched as much as possible under the microscope. The LMMP was washed three times with $4 \mathrm{ml}$ of ice-cold PBS and Dulbecco's modified Eagle's medium. Immediately thereafter, the LMMP was placed in Dulbecco's modified Eagle's medium (Gibco, Foster City, CA) containing collagenase II $(1 \mathrm{mg} / \mathrm{ml}$, Gibco), cut into pieces with scissors, and digested in a humidified incubator (Thermo Forma, Hamilton, USA) with 5\% $\mathrm{CO}_{2}$ at $37{ }^{\circ} \mathrm{C}$ for $55 \mathrm{~min}$. After digestion, the medium was added to terminate and centrifuged at $1000 \mathrm{RPM}$ for $6 \mathrm{~min}$. To culture enteric neurons, the sediment was 
collected and suspended in Dulbecco's modified Eagle's medium (Gibco, Foster City, CA) supplemented with 10\% heat-inactivated foetal bovine serum (Gibco, Foster City, CA) and $1 \%$ penicillin-streptomycin solution (Gibco). The medium was replaced with fresh medium daily, and the cell growth status was carefully observed. On day 2 after digestion, we added cytarabine (Gibco, $5 \mu \mathrm{M}$ ) to the culture to inhibit the growth of glial cells and miscellaneous cells, and then pure cultured primary neurons were obtained.

\section{Experimental animals}

Wild-type C57BL6/J mice were purchased from Beijing Vital River Laboratory Animal Technology and housed in a specific pathogen-free environment in the Animal Center of Shandong University. All mice in the experiment were aged 7-10 weeks and were randomly grouped. All experiments were performed at the Animal Center of Shandong and approved by the Medical Ethics Committee for Experimental Animals, Shandong University School of Basic Medicine Sciences (ECSBMSSDU2020-2-006). All measures were taken to minimize animal suffering.

\section{Immunocytofluorescence}

The 24-well plate cell slides (coated with polylysine) or the LMMP were washed twice with cold PBS and were regularized with $4 \%$ paraformaldehyde at room temperature for $20 \mathrm{~min}$, then washed three times with PBS for $15 \mathrm{~min}$. The cell slides or the LMMP were handled with $3 \%$ hydrogen peroxide for $10 \mathrm{~min}$ and washed three times with PBS for $15 \mathrm{~min}$. The cell slides or the LMMP were handled with $0.3 \%$ Triton-X100 for $60 \mathrm{~min}$ and washed three times with PBS for 15 min. After sealed with $10 \%$ donkey serum for $60 \mathrm{~min}$ at room temperature, immediately the cell slides or the LMMP were incubated with mouse anti-tubulin (1:800, Abcam), rabbit anti-OT (1:200, Invitrogen) primary antibodies diluted in blocking solution overnight at $4^{\circ} \mathrm{C}$.

Paraffin sections of mouse colons were dewaxed for $2 \mathrm{~h}$, soaked in xylene for $5 \mathrm{~min}$, soaked in 100\% alcohol, 95\% alcohol, 90\% alcohol, 75\% alcohol, and 50\% alcohol for $3 \mathrm{~min}$ each, and then rinsed with water for $3 \mathrm{~min}$. Then, antigen retrieval was performed by boiling the slices in sodium citrate buffer (10 mM, pH 6, Beyotime) for $25 \mathrm{~min}$. After cooling, the tissues were blocked with $10 \%$ donkey serum for $50 \mathrm{~min}$ at $25^{\circ} \mathrm{C}$ and then incubated with mouse anti-Iba1(1:200, Abcam), mouse antitubulin (1:800, Abcam), rabbit anti-Arg1(1:200, Abcam), rabbit anti-F4-80 (1:200, Abcam) and mouse anti-iNOS (1:200, Abcam) primary antibodies diluted with blocking solution overnight at $4{ }^{\circ} \mathrm{C}$. After three washes, the slides were incubated with Alexa Fluor 568 or 488 donkey anti-rabbit (1:1000, Invitrogen) or Alexa Fluor 488 donkey anti-mouse (1:1000, Invitrogen) secondary antibodies for $60 \mathrm{~min}$, followed by counterstaining with DAPI (1:1000, Beyotime) for $5 \mathrm{~min}$. Images were captured by a Laser Confocal Microscope (LSM980).

\section{RNA extraction and quantitative real-time PCR}

Total RNA was extracted from neurons and LMMP by a tissue/cell rapid extraction kit (Spark jade, Qingdao, China). In short, lysis solution RL was added to lyse the cells and tissue fluid after homogenization to depolymerize and release protein and nucleic acid substances. Next, chloroform, absolute ethanol, protein-removing solution $\mathrm{RE}$ and rinsing solution RW were added in sequence to obtain approximately $20 \mu$ of RNA solution. RNA was reverse-transcribed in a Takara PCR Thermal Cycler SP (Takara Bio, Shiga, Japan). Real-time quantitative PCR was carried out with SYBR Premix Dimer Eraser (Takara Bio, Shiga, Japan). Expression levels were normalized to the internal controls ( $\beta$-actin), and the relative expression level was evaluated using the $2^{-\triangle \triangle \mathrm{CT}}$ method. The primers were designed and verified by the Shanghai Genomics Institute (Shanghai, China), and the sequences of the primers are shown in Table 1.

\section{ELISA}

To assess levels of IL- $1 \beta$, TNF- $\alpha$, IL- 6 , TGF- $\beta$, and OT, enteric neuron and tissue supernatants were collected and centrifuged, and the concentrations of a specific protein were measured using a precoated ELISA kit according to the instruction manual.

\section{Colon explant culture}

Colons from naive or colitis mice were flushed 10 times with PBS to remove faeces. Two 5-mm-long segments of colon tissue were cultured in $250 \mu \mathrm{l}$ of RPMI supplemented with $10 \%$ heat-inactivated foetal bovine serum, $1 \%$ penicillin-streptomycin and $50 \mathrm{mg} / \mathrm{ml}$ gentamycin (Wako) in a 48-well plate (Corning, NY) at $37{ }^{\circ} \mathrm{C}$ for $30 \mathrm{~min}$ [24]. OT in the culture supernatant was measured by an ELISA kit.

\section{Western blotting analysis}

Enteric neurons and tissues were harvested in ice-cold RIPA buffer (Bioster Bio, Pleasanton, CA) containing $1 \%$ PMSF and $1 \%$ phosphatase inhibitor (Bioster Bio, Pleasanton, CA). After stimulation with specific drugs, the cells were centrifuged at $13,800 \times g / 10 \mathrm{~min}$ at $4{ }^{\circ} \mathrm{C}$. The supernatant was retained, and a $\mathrm{BCA}$ protein assay kit (Boster, USA) was used to quantify the total protein concentration. Next, the samples were mixed with $5 \times$ loading buffer and boiled for $10 \mathrm{~min}$ at $100^{\circ} \mathrm{C}$. Then, the proteins were loaded onto $15 \%, 10 \%$ and $8 \%$ gradient 
Table 1 The primer sequences used in the study as follows

\begin{tabular}{|c|c|}
\hline Name & Primer sequence $\left(5^{\prime} \rightarrow 3^{\prime}\right)$ \\
\hline \multirow[t]{2}{*}{$\beta$-actin } & F primer: CTATTGGCAACGAGCGGTTCC \\
\hline & R primer: CAGCACTGTGTTGGCATAGAG \\
\hline \multirow[t]{2}{*}{ TNFa } & F primer: CCCTCACACTCAGATCATCTTCT \\
\hline & R primer: GCTACGACGTGGGCTACAG \\
\hline \multirow[t]{2}{*}{ IL6 } & F primer:TCCTTCCTACCCCAATTCCA \\
\hline & R primer: GTCTTGGTCCTTAGCCACTCC \\
\hline \multirow[t]{2}{*}{ ILI $\beta$} & F primer: GGCAACCGTACCTGAACCCA \\
\hline & R primer: CCACGATGACCGACACCACC \\
\hline \multirow[t]{2}{*}{ iNOS } & F primer: CCGAAGCAAACATCACATTCA \\
\hline & R primer: GGTCTAAAGGCTCCGGGCT \\
\hline \multirow[t]{2}{*}{ CCL2 } & F primer: TTAAAAACCTGGATCGGAACCAA \\
\hline & R primer: GCATTAGCTTCAGTTACGGGT \\
\hline \multirow[t]{2}{*}{$\operatorname{Arg} 1$} & F primer:TGTCCCTAATGACAGCTCCTT \\
\hline & R primer: GCATCCACCCAAATGACACAT \\
\hline \multirow[t]{2}{*}{ ChIL3 } & F primer: GATGGCCTCAACCTGGACTG \\
\hline & R primer: CGTCAATGATTCCTGCTCCT \\
\hline \multirow[t]{2}{*}{ CD206 } & F primer:TGATTACGAGCAGTGGAAGC \\
\hline & R primer: GCTACGACGTGGGCTACAG \\
\hline \multirow[t]{2}{*}{ OTR } & F primer: GGCCGTGTTCCAGGTTCTC \\
\hline & R primer:TGCAAGTATGACCAGACGAC \\
\hline \multirow[t]{2}{*}{ OT } & F primer: ATCACCTACAGCGGATCTCAGAC \\
\hline & R primer: CAGAGCCAGTAAGCCAAGCA \\
\hline \multirow[t]{2}{*}{ IL10 } & F primer:TAACTGCACCCACTTCCCAG \\
\hline & R primer: TTGGCAACCCAAGTAACCCTTA \\
\hline \multirow[t]{2}{*}{ TGF $\beta$} & F primer: AGCTGCGCTTGCAGAGATTA \\
\hline & R primer: AGCCCTGTATTCCGTCTCCT \\
\hline \multirow[t]{2}{*}{ Ym1 } & F primer: AGAAGGGAGTTTCAAACCTGGT \\
\hline & R primer: GTCTTGCTCATGTGTGTAAGTGA \\
\hline
\end{tabular}

polyacrylamide gels and electrophoretically transferred to polyvinylidene difluoride membranes. The membranes were blocked with $5 \%$ nonfat dry milk for $90 \mathrm{~min}$ at $25{ }^{\circ} \mathrm{C}$ and then incubated with the following primary antibodies overnight at $4{ }^{\circ} \mathrm{C}$ : anti-OT $(1: 400$, Invitrogen) antibody, anti-OTR (1:800, Abcam) antibody, antiPEG3 (1:800, Abcam) antibody, anti-p65 (1:1000, CST) antibody, anti-phospho-p65 (1:800, CST) antibody, antiSTAT3 (1:1000, CST) antibody, anti-phospho-STAT3 (1:1000, CST) antibody, phospho-SMAD2 (1:400, CST) antibody, anti-SMAD2 (1:1000, CST) antibody, phosphoSMAD3 (1:500, CST) antibody, anti-SMAD3 (1:1000, CST) antibody and anti- $\beta$-actin (1:2000, Zhongshan Golden Bridge Biotechnology) antibody. After multiple washes, the membranes were incubated with secondary antibodies (1:2000, Beyotime) at room temperature for $50 \mathrm{~min}$. The ECL kit reagent (MILLIPORE, USA) was used to generate chemiluminescence signals, which were captured with the Tanon Imaging System (Tanon-4600). The signal strength was analysed by ImageJ software.

\section{DSS colitis model}

Acute colitis was induced by $2.5 \%$ DSS salt (reagentgrade, MW 36-50 kDa, MP Biomedicals, Canada) in drinking water for 7 days. In the second week, standard drinking water was available to the mice. The mice were randomly allocated into six groups: (1) control, (2) 1 W-DSS, (3) 1 W-DSS + 1 W-WATER, (4) $1 \mathrm{~W}$-DSS + 2 W-WATER, (5) $1 \mathrm{~W}$-DSS + 3 W-WATER, (6) $1 \mathrm{~W}-\mathrm{DSS}+6 \mathrm{~W}-\mathrm{WATER}$. Mice were euthanized by intraperitoneal administration of sodium pentobarbital $(200 \mathrm{mg} / \mathrm{kg})$ on days $7,14,21,28$ and 49 . The distal colon was embedded in $4 \%$ paraformaldehyde solution, and the transverse sections were stained with haematoxylin and eosin. Histological scores were determined independently by two analysts who were blinded to the animal groupings. The assessment criteria included epithelial surface damage, crypt loss and inflammatory infiltrate as described by Zaki MH. The final score ranged from 0 to 6 (combining the inflammatory cell infiltration score and tissue damage score).

\section{D-Mannose model}

Mice were fed 20\% D-mannose (Sigma-Aldrich, St. Louis, MO) for 7 days $[25,26]$. Clodronate liposomes (FormuMax, Silicon Valley, CA) were injected intraperitoneally one day before initiating the model and the third day after initiating the model to deplete macrophages; control liposomes were used as the control group [27-29]. The mice were randomly allocated into six groups: (1) control, (2) control + D-mannose, (3) control liposomes, (4) control liposomes +D-mannose, (5) clodronate liposomes, (6) clodronate liposomes + D-mannose. Distal colons were embedded in $4 \%$ paraformaldehyde solution, and transverse sections were stained with haematoxylin and eosin.

\section{Statistical analysis}

Data are expressed as the mean \pm SEM from at least two independent experiments. The number of samples in a specific experiment is reported. One-way ANOVA or two-tailed Student's $t$-test was used for comparisons between groups. GraphPad Prism version 5 (La Jolla, CA) was used for statistical analysis. $P<0.05$ was considered statistically significant.

\section{Results}

Effect of macrophage polarization on the OT signalling system

To investigate the effect of macrophage polarization on the OT signalling system, we determined the position of OT in enteric neurons and further confirmed that OT was expressed in the bodies and nerve fibres 
A

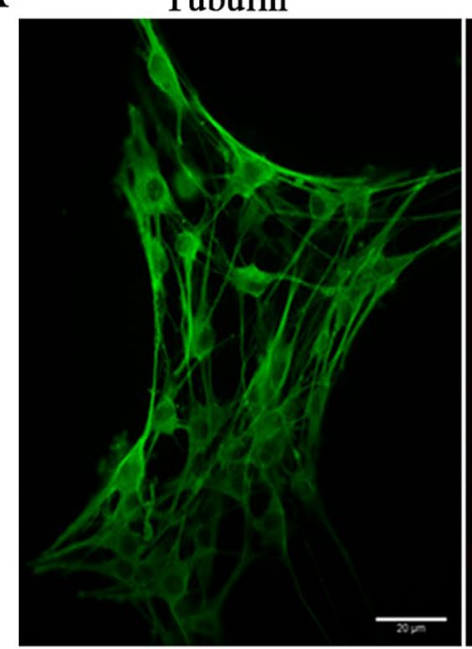

B

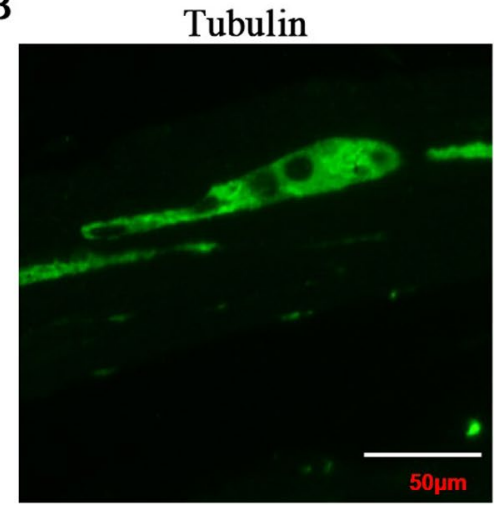

Oxytocin

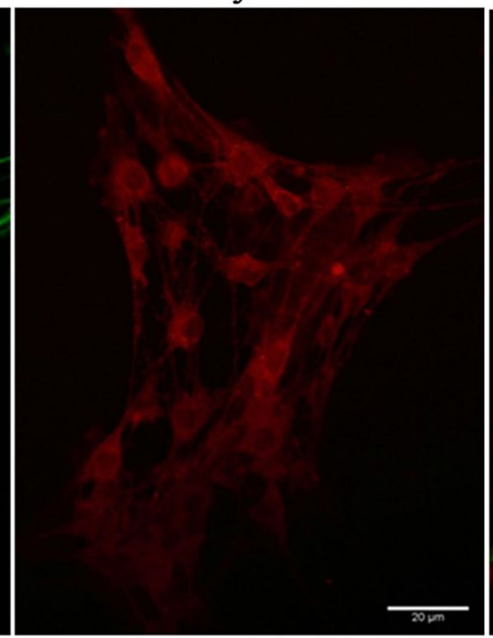

Oxytocin

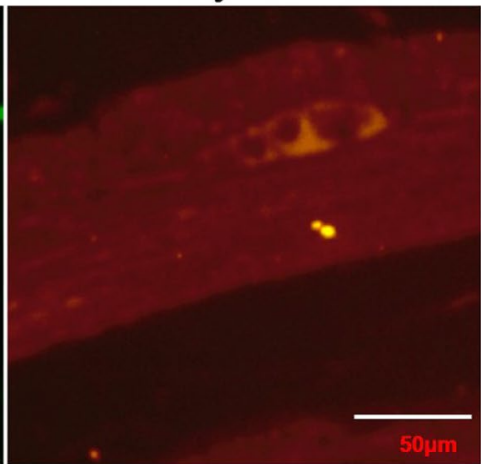

Merge

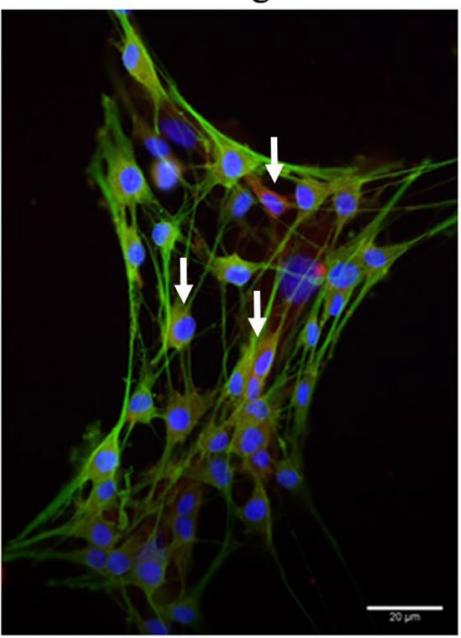

Merge

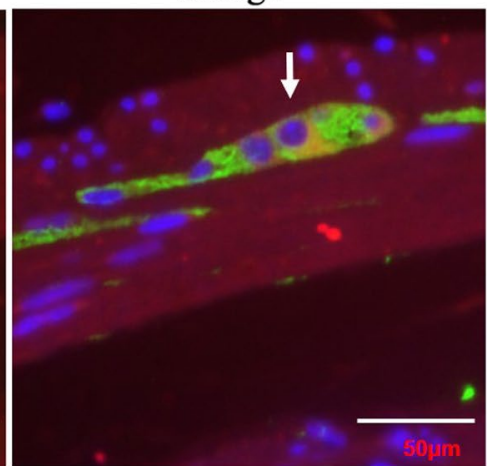

Fig. 1 OT is mainly expressed in neuron bodies and nerve fibres. A Representative photographs of double immunofluorescent staining of tubulin (green) and oxytocin (OT) (red) within randomly captured images were obtained in cultured enteric neurons. Scale bar $=20 \mu \mathrm{m}$. B Representative photographs of double immunofluorescent staining of Tubulin (green) and Oxytocin (OT) (red) within randomly captured images were obtained in colon cross sections. Scale bar $=50 \mu \mathrm{m}$

(Fig. 1A, B). We used LPS or IL-4 to stimulate enteric neurons and observed no obvious changes in OT or OTR mRNA expression or OT secretion compared with the control group (Additional file 1: Fig. S1A-C). We used LPS to induce RAW264.7 cells to differentiate into M1 macrophages and collected the supernatant (M1 supernatant). We also induced RAW264.7 cells to differentiate into the M2 type with IL4 and collected the supernatant (M2 supernatant). We separately incubated enteric neurons with M1 supernatant, M2 supernatant or unstimulated macrophages supernatant (M0 supernatant) (Fig. 2A). In cells treated with M1 supernatant, the mRNA levels of OT and OTR were markedly decreased compared with those in the control group (Fig. 2G), whereas treatment with the M2 supernatant increased the mRNA expression of OT and OTR (Fig. 2H). This result was also verified in enteric neurons extracted from female mice (Additional file 1: Fig. S1E). Results from staining indicated that M1 supernatant inhibited the expression of OT, and M2 supernatant promoted the expression of OT compared with M0 supernatant in enteric neurons (Fig. 2F). The effects of the macrophage supernatant on OT and OTR protein expression were similar to those on mRNA expression (Fig. 2B, C, D, E, I).

\section{Effect of cytokines in macrophages supernatant} on the expression of OT and OTR in enteric neurons

To investigate cytokines in M1 or M2 macrophages supernatant that regulate the expression of OT and OTR, we first measured the levels of different cytokines in the supernatant. The levels of TNF- $\alpha$, IL- 6 and IL- $1 \beta$ were obviously increased in M1 supernatant (Fig. 3A-C), whereas in M2 supernatant, TGF- $\beta$ levels were obviously increased (Fig. 3D). Second, we tested the effects of these cytokines on the mRNA expression of OT and OTR. Treatment with IL- 6 , TNF- $\alpha$ or IL- $1 \beta$ significantly 


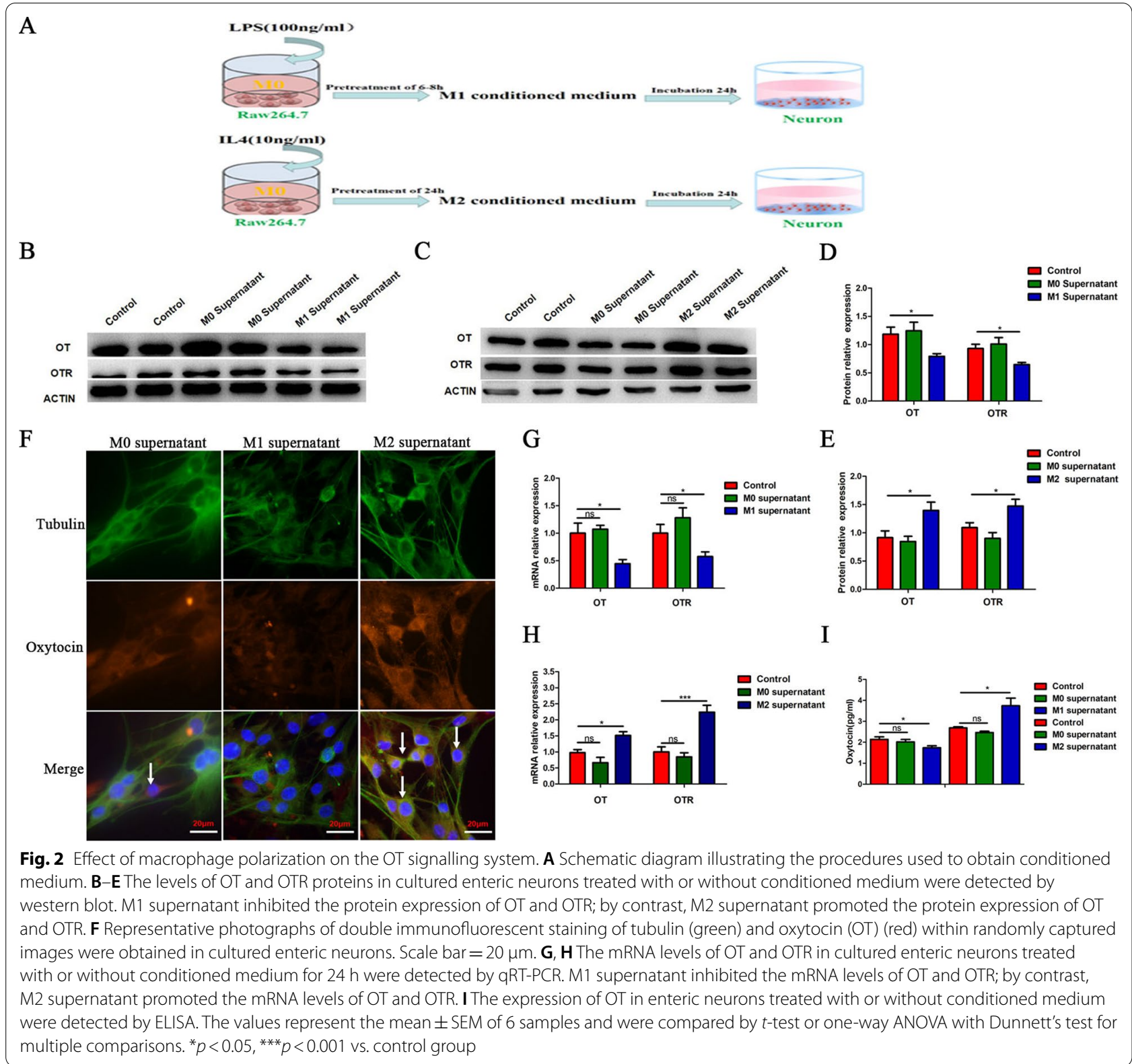

decreased the mRNA expression of OT and OTR in cultured enteric neurons (Fig. 3E-G) and reduced the release of OT in cultured enteric neurons (Fig. 3J). By contrast, TGF- $\beta$ increased the mRNA levels of OT and OTR in cultured enteric neurons (Fig. 3I), and IL-10 seemed to have no effect (Fig. 3H).

To test whether the above cytokines affected the expression of OT and OTR through their receptors, we pretreated neurons with tocilizumab (IL-6 receptor antagonist), R-7050 (TNF-a receptor antagonist), or LY2109761 (TGF- $\beta$ antagonist). We found that tocilizumab, R-7050 and LY2109761 reversed the inhibitory (IL- 6 and TNF- $\alpha$ ) and excitatory (TGF- $\beta$ ) effects of the respective agonists (Fig. 4A-C, Additional file 1: Fig. S1D). In addition, treatment with IL-1Ra, tocilizumab and R-7050 completely reversed the excitatory effect of M1 supernatant on the expression of OT, and LY2109761 completely reversed the inhibitory effect of M2 supernatant (Fig. 4D, E). Therefore, we believe that the inhibitory effect of M1 supernatant on OT and OTR expression occurs mainly through IL- $1 \beta$, IL- 6 and TNF- $\alpha$, whereas the excitatory effect of M2 supernatant originates mainly from TGF- $\beta$. 


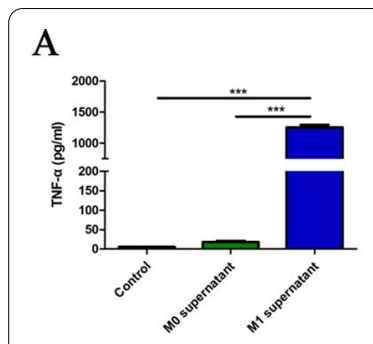

$\mathrm{E}$

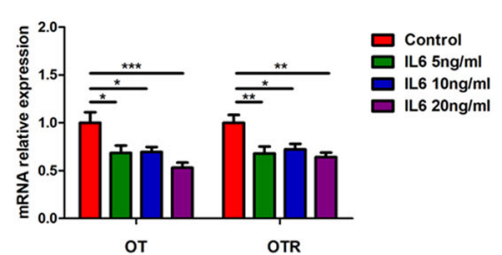

$\mathrm{H}$

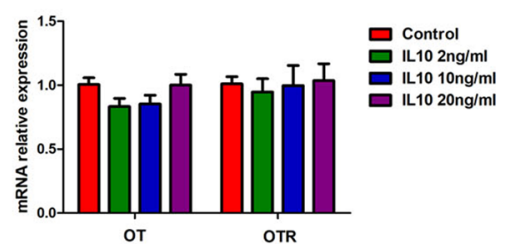

B

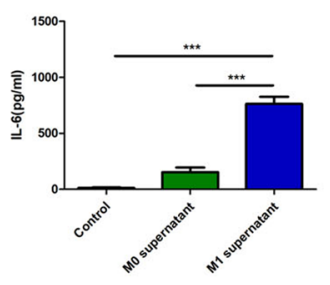

F
C

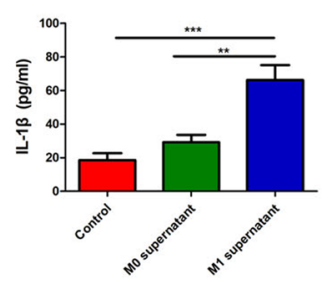

D

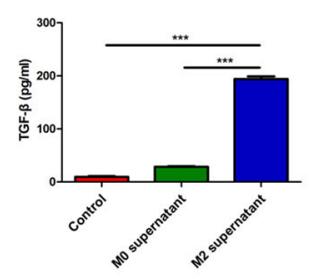

G

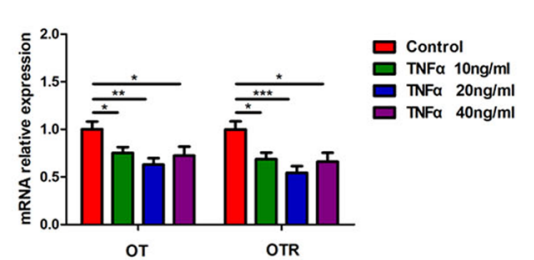

I

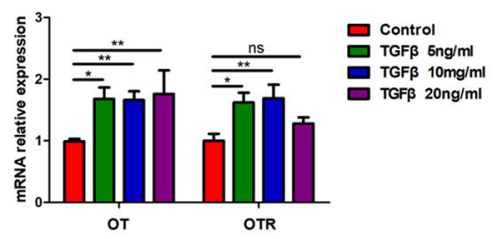

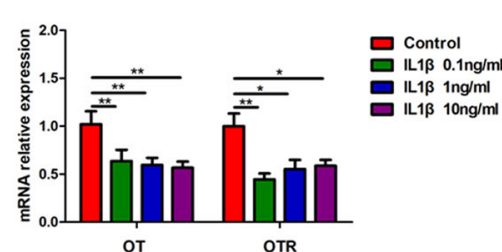

J

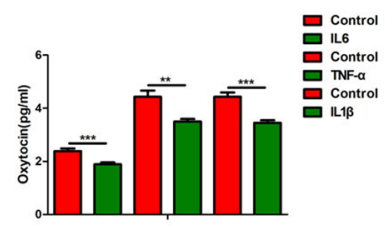

Fig. 3 Effect of cytokines in macrophages supernatant on the expression of OT and OTR in enteric neurons. A-C M1 supernatant treatment for $24 \mathrm{~h}$ increased IL-6, TNF- $a$ and IL-1 $\beta$ levels in cultured enteric neurons as assessed by ELISA. D M2 supernatant treatment for $24 \mathrm{~h}$ increased TGF- $\beta$ levels in cultured enteric neurons as assessed by ELISA. E-G Stimulation of cultured enteric neurons for $6 \mathrm{~h}$ with IL-6 $(0,5,10,20 \mathrm{ng} / \mathrm{ml})$, TNF- $\alpha(0$, $10,20,40 \mathrm{ng} / \mathrm{ml})$ or IL-1 $\beta(0,0.1,1,10 \mathrm{ng} / \mathrm{ml})$ decreased the mRNA expression of OT and OTR. $\mathbf{H}$ Stimulation of cultured enteric neurons with IL $10(0$, $2,10,20 \mathrm{ng} / \mathrm{ml})$ did not affect the expression of OT and OTR mRNA. I Stimulation of cultured enteric neurons with TGF- $\beta$ (0, 5, 10, $20 \mathrm{ng} / \mathrm{ml})$ for $6 \mathrm{~h}$ increased the mRNA expression of OT and OTR. J The concentration of OT was measured in cultured enteric neurons treated with or without IL-6 $(10 \mathrm{ng} / \mathrm{ml})$, TNF- $\mathrm{a}(20 \mathrm{ng} / \mathrm{ml})$, and IL-1 $\beta(1 \mathrm{ng} / \mathrm{ml})$ for $30 \mathrm{~min}$ by ELISA. The values represent the mean \pm SEM of 6 samples and were compared by one-way ANOVA with Newman-Keuls and Dunnett's test for multiple comparisons. ${ }^{*} p<0.05,{ }^{* *} p<0.01,{ }^{* * *} p<0.001$

\section{Inflammatory cytokines downregulate the expression of OT/OTR proteins in enteric neurons via the STAT3 or NF-kB pathway}

Inflammatory factors can activate the STAT3 [30] and NF- $\mathrm{kB}$ pathways to participate in intracellular reactions $[22,31]$. We therefore investigated whether these two pathways were involved in the downregulation of the protein expression of OT and OTR following IL-6, TNF- $\alpha$ and IL- $1 \beta$ treatment. The degree of STAT3 phosphorylation was significantly higher following treatment with IL- 6 or TNF- $\alpha$ for $24 \mathrm{~h}$ than in the control group (Fig. 5A, $\mathrm{C}, \mathrm{E}, \mathrm{G})$. In addition, the protein expression of OT and OTR decreased significantly, and this inhibitory effect was reversed by stattic (a STAT3 inhibitor, $5 \mu \mathrm{M}$ ) (Fig. 5B, $\mathrm{D}, \mathrm{F}, \mathrm{H})$. Treatment with IL- $1 \beta$ did not change the degree of STAT3 phosphorylation (Additional file 1: Fig. S1F), but significantly upregulated the protein expression of $\mathrm{p}-\mathrm{NF}-\mathrm{kB}$ in enteric neurons (Fig. 5I, K). The decreases in OT and OTR expression following IL-1 $\beta$ administration were significantly reversed by pretreatment with
Bay11-7082 (an NF-kB inhibitor, $1 \mu \mathrm{M}$ ) (Fig. 5J, L). Therefore, we believe that the inflammatory cytokines in M1 macrophages supernatant inhibit the expression of OT and OTR via different pathways. TNF- $\alpha$ and IL- 6 may elicit their effects through STAT3 phosphorylation, whereas the influence of IL- $1 \beta$ is mainly related to NF- $\mathrm{kB}$ phosphorylation.

\section{The Smad2/3 pathway is involved in the upregulation of OT/OTR expression following TGF- $\beta$}

TGF- $\beta$ induces a cellular response by binding transmembrane receptors [32] and phosphorylating Smad2/3 (receptor regulated Smads: R-Smads) [33]. In this study, we found that $\mathrm{p}-\mathrm{Smad} 2$ and $\mathrm{p}-\mathrm{Smad} 3$ expression were upregulated following TGF- $\beta$ treatment for $24 \mathrm{~h}$, and pretreatment with SIS3CHL (Smad3 inhibitor, $5 \mu \mathrm{M}$ ) blocked this change (Fig. 6A-D). Therefore, we believe that TGF- $\beta$ increases OT and OTR expression in enteric neurons via the Smad2/3 pathway. 


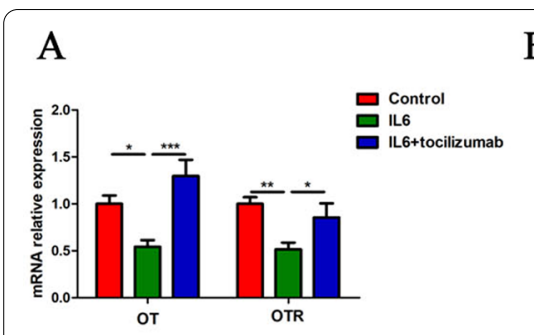

$\mathrm{D}$

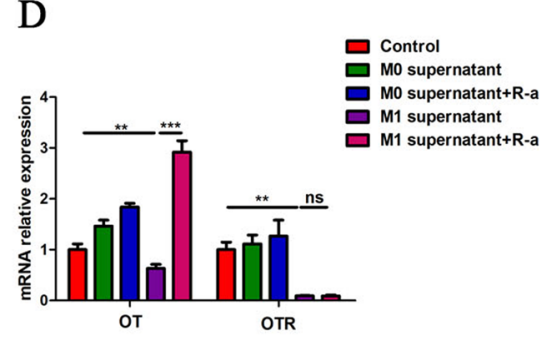

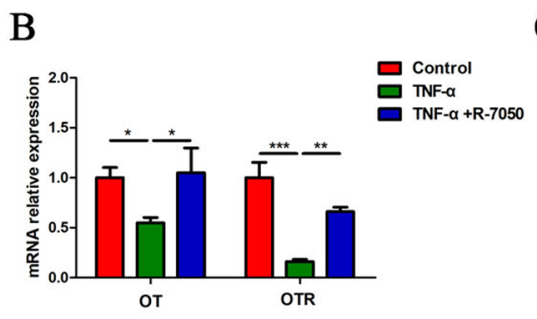

$\mathrm{E}$

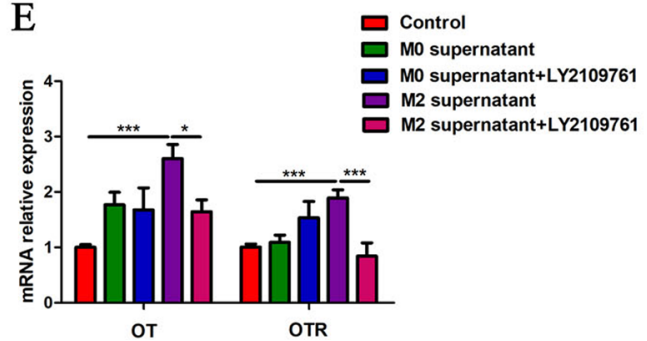

Fig. 4 Effects of cytokine receptor antagonistson OT and OTR expression in cultured enteric neurons. A Cultured enteric neurons were pretreated with tocilizumab (IL-6-Ra, $1 \mu \mathrm{M})$ for $12 \mathrm{~h}$ followed by $\mathrm{lL}-6(10 \mathrm{ng} / \mathrm{ml})$ for $6 \mathrm{~h}$. The relative mRNA expression levels of OT and OTR were analysed by qRT-PCR. B Cultured enteric neurons were pretreated with R-7050 (TNF-a receptor antagonist, $1 \mu \mathrm{M}$ ) for $12 \mathrm{~h}$ followed by TNF-a (20 ng/ml) for $6 \mathrm{~h}$. The relative mRNA expression levels of OT and OTR were analysed by qRT-PCR. C Cultured enteric neurons were pretreated with LY2109761 (TGF- $\beta$ receptor antagonist, $1 \mu \mathrm{M}$ ) for $12 \mathrm{~h}$ followed by TGF- $\beta(10 \mathrm{ng} / \mathrm{ml})$ for $6 \mathrm{~h}$. The relative mRNA expression levels of OT and OTR were analysed by qRT-PCR. D Cultured enteric neurons were pretreated with tocilizumab, R-7050 or IL-1 ra for $12 \mathrm{~h}$ followed by M0 and M1 supernatant for $24 \mathrm{~h}$. The relative expression levels of OT and OTR mRNA were analysed by qRT-PCR. Notably, the three receptor antagonists reversed the effect of M1 supernatant on the expression of OT. E Cultured enteric neurons were pretreated with LY2109761 for $12 \mathrm{~h}$ followed by M0 or M2 supernatant for $24 \mathrm{~h}$. The relative expression levels of OT and OTR mRNA were analysed by qRT-PCR. LY2109761 downregulated the mRNA expression of OT and OTR by inhibiting the expression of TGF- $\beta$. Values represent the mean \pm SEM of 6 samples and were compared by one-way ANOVA with Newman-Keuls and Dunnett's test for multiple comparisons. ${ }^{*} p<0.05,{ }^{* *} p<0.01,{ }^{* * *} p<0.001$

\section{Relationship between macrophage polarization and OT and OTR expression in DSS-induced colitis}

Our laboratory has previously shown that the OT signalling system reduces intestinal inflammation by regulating the polarization of macrophages in experimental colitis $[10,22,34]$. In this study, we explored whether the expression of the OT signalling system was impacted by DSS-induced colitis (Additional file 2: Fig. S2A). In the first 7 days during which the mice were administered DSS in drinking water, the mice in the DSS treatment group had significantly reduced body weight, reduced colon length, and a higher disease activity index than those in the control group (Additional file 2: Fig. S2B-E). These changes reached a maximum at the end of the first week after the beginning of DSS feeding and then began to decrease. At the third week, the weight of the mice and the colon length returned to normal, and the disease activity index decreased significantly (Additional file 2: Fig. S2B-E). Histological evaluation at different time periods showed that at 7 days following DSS treatment, the colon exhibited severe epithelial damage, crypt loss, submucosal oedema, and inflammatory cell infiltration. However, these histological changes began to decrease at the second week and completely disappeared at the seventh week (Additional file 2: Fig. S2F, G). Therefore, we divided DSS-induced colitis into the inflammatory phase (the first and second weeks) and the recovery phase (the third week and later) (Additional file 2: Fig. S2A). The previous data indicated that M1 supernatant, IL-1 $\beta$, TNF- $\alpha$, and IL- 6 inhibited the mRNA expression levels of OT and OTR in cultured enteric neurons, whereas M2 supernatant and TGF- $\beta$ promoted their expression (Figs. 2, 3, 4). In the inflammatory phase of DSS-induced colitis, IL-1 $\beta$, TNF- $\alpha$ and IL- 6 mRNA expression were significantly increased in the colon (Fig. 7A-C). In addition, the mRNA expression of the M1 macrophages markers CCL2 and iNOS were significantly increased (Fig. 7D). The number of M1 phenotypes (F4-80 + iNOS ${ }^{+}$) was significantly increased (Fig. 8). These parameters reached their highest levels at the first or second week and gradually decreased to normal in the recovery period. By contrast, the mRNA expression of TGF- $\beta$ and the M2 macrophage markers CD206, Arg1, and Chil3 did not change in the first week but began to increase at the second week and reached the highest level at the third or fourth week (Fig. 7E-G). The changes in the concentrations of IL- 6 , IL- $1 \beta$, TNF- $\alpha$ and TGF- $\beta$ showed similar dynamics (Fig. 7H, I). Therefore, we believe that macrophages are polarized to the M1 type during the inflammatory phase and to the M2 type during the recovery 

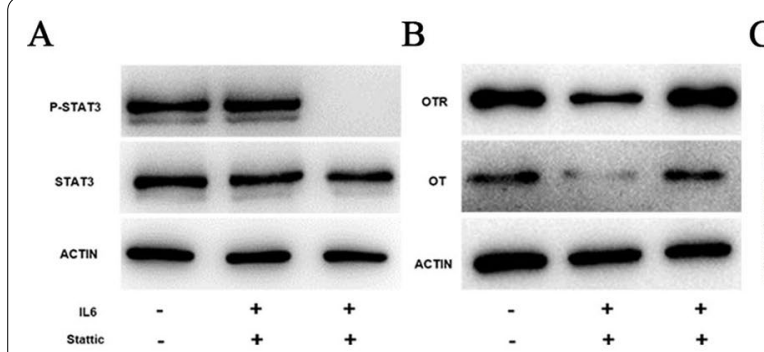

E

F

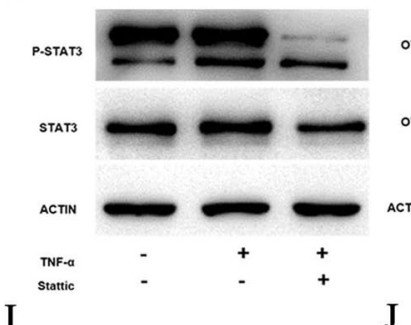

J

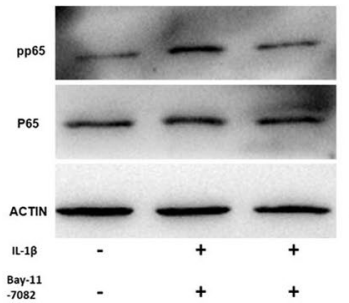

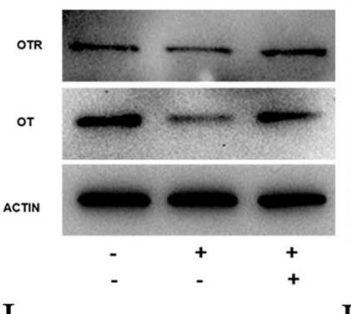

G

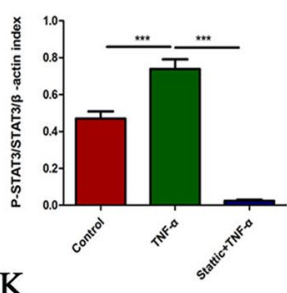

K

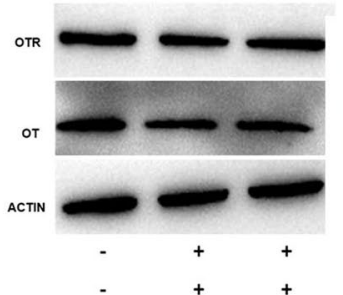

$\mathrm{C}$

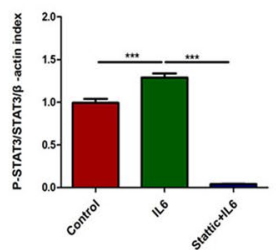

D
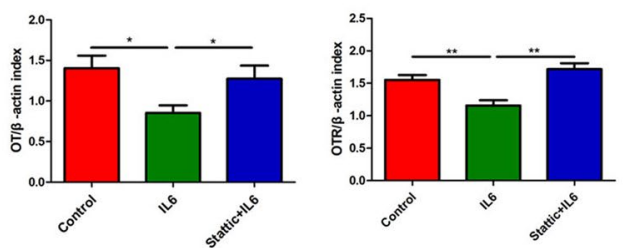

$\mathrm{H}$
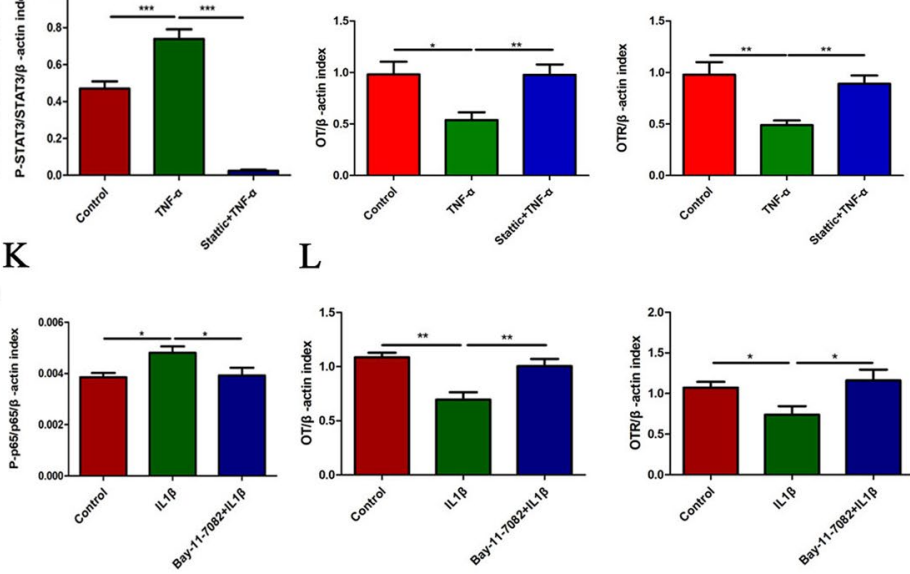

L

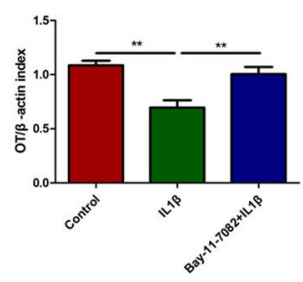

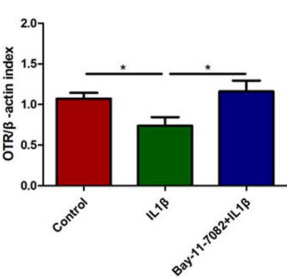

Fig. 5 Inflammatory cytokines downregulate the protein expression of OT/OTR in enteric neurons via the STAT3 or NF-KB pathway. A-D Cultured enteric neurons were pretreated with or without Stattic (STAT3 inhibitor, $5 \mu \mathrm{M}$ ) for $12 \mathrm{~h}$ followed by IL6 (10 ng/ml) or TNF-a (20 ng/ml) for $24 \mathrm{~h}$. The levels of phosphorylated STAT3 (p-STAT3), total STAT3, OT, and OTR were analysed by western blot, and $\beta$-actin was used to evaluate protein loading. IL-6 $(10 \mathrm{ng} / \mathrm{ml})$ or TNF- $\mathrm{a}(20 \mathrm{ng} / \mathrm{ml})$ decreased the expression of OT and OTR by activating the STAT3 pathway. E, F Cultured enteric neurons were pretreated with or without Bay 11-7082 (NF-KB inhibitor, $1 \mu \mathrm{M}$ ) for $2 \mathrm{~h}$ followed by IL-1 $\beta$ (1 ng/ml) for $24 \mathrm{~h}$. The levels of phosphorylated NF-KB (p-p65), total NF-KB (p65), OT, and OTR were analysed by western blot, and $\beta$-actin was used to evaluate protein loading. IL-1 $\beta$ (1 ng/ml) decreased the expression of OT and OTR by activating the NF-KB pathway. Values represent the mean \pm SEM of 6 samples and were compared by one-way ANOVA with Newman-Keuls for multiple comparisons. ${ }^{*} p<0.05,{ }^{* *} p<0.01,{ }^{* * *} p<0.001$

phase. Next, we assessed the expression of the OT signalling system in the two phases in DSS-treated mice. The mRNA expression of OT and OTR decreased significantly during the inflammatory phase and increased significantly during the recovery phase (Fig. 7J). The level of OT in colon explant and the density of neurons in the each ganglion showed the same dynamic changes (Fig. 7K and Additional file 3: Fig. S3). We believe that the expression of the OT signalling system and macrophage polarization in the colon are correlated in these two phases of DSS-induced colitis.

\section{M2 macrophage polarization increases the expression of OT and OTR in the colon}

To further test the hypothesis that M2 macrophage polarization increases OT and OTR expression in the gut, we used D-mannose (a commonly used glyconutrient in clinical practice) to activate macrophages [35]. The mice were fed D-mannose for 7 days. The body weight of the mice decreased on the second day and began to recover on the fourth day (Additional file 4: Fig. S4A). There were no changes in eating habits and the histological structure of the colon (Additional file 4: Fig. S4B, C), but the size and weight of the caecum and colon increased (Additional file 4: Fig. S4D). We also confirmed those results in female mice (Additional file 5: Fig. S5A). D-Mannose has been reported to inhibit M1 polarization [35]. After 7 days of D-mannose feeding, iNOS mRNA expression in the colon was decreased, but the mRNA expression of IL-6, IL- $1 \beta$ and TNF- $\alpha$ did not change (Additional file 4: Fig. S4F). Surprisingly, the mRNA expression of surface markers of M2 macrophages in the colon, including CD206, Arg1, Ym1 and Chil3, significantly increased, and the anti-inflammatory factors TGF- $\beta$ and IL10 were also significantly upregulated (Additional file 4: Fig. S4E). Therefore, we believe that D-mannose promotes the polarization of M2 macrophages and inhibits the polarization of M1 macrophages. Consistent with the change in 

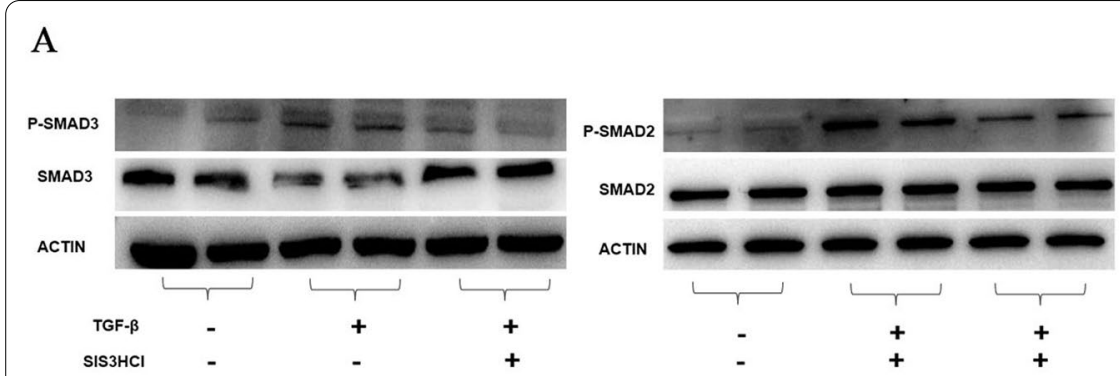

B

C
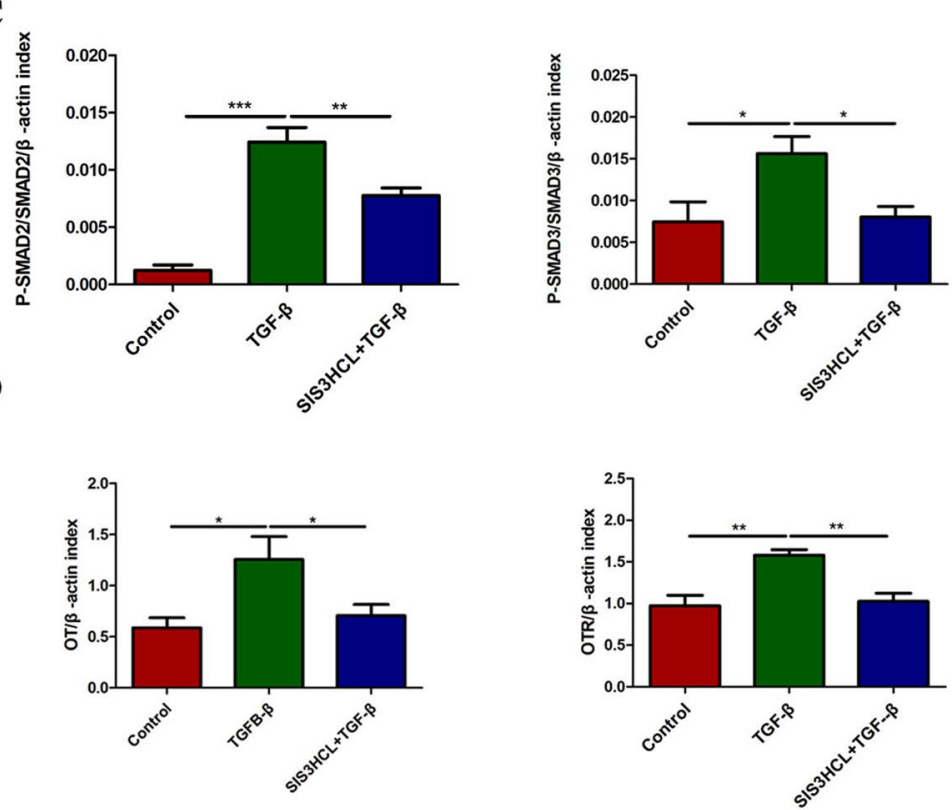

Fig. 6 The Smad2/3 pathway is involved in the upregulation of OT/OTR expression following TGF- $\beta$ treatment. A-D Cultured enteric neurons were pretreated with or without SIS3CHL (Smad3 inhibitor, $5 \mu \mathrm{M}$ ) for $12 \mathrm{~h}$ followed by TGF- $\beta(10 \mathrm{ng} / \mathrm{ml}$ ) for $24 \mathrm{~h}$. The levels of phosphorylated Smad2 ( $p$-Smad2), phosphorylated Smad3 (p-Smad3), total Smad2, total Smad3, OT, and OTR were analysed by western blot, and $\beta$-actin was used to evaluate protein loading. TGF- $\beta(10 \mathrm{ng} / \mathrm{ml})$ increased the expression of OT and OTR by activating the $\mathrm{Smad} 2 / 3$ pathway. The values represent the mean \pm SEM of 6 samples and were compared by one-way ANOVA with Newman-Keuls for multiple comparisons. ${ }^{*} p<0.05,{ }^{* *} p<0.01,{ }^{* * *} p<0.001$

macrophage polarization, OT and OTR expression were also significantly upregulated (Additional file 4: Fig. S4G). We also confirmed those results in female mice (Additional file 5: Fig. S5B).

To test the hypothesis that the increase in the OT signalling system following D-mannose treatment is attributable to M2 polarization, we depleted macrophages via systemic administration of clodronate liposomes [29, 36-38] (Fig. 9A) and observed the expression of the OT signalling system. After clodronate liposome pretreatment, the number of Iba1/Arg1 double-positive cells (Fig. 9E, left), CD206-positive cells (Fig. 9E, right) and the expression of M1 and M2 macrophage surface markers (Fig. 9F) were significantly reduced. Treatment with clodronate liposomes decreased body weight, but did not influence the eating and drinking habits of the mice nor the size and weight of the colon and caecum (Fig. 9B, C and Additional file 4: Fig. S4I, J). Pretreatment with clodronate liposomes significantly reversed the upregulation of OT and OTR expression in the colon induced by systemic administration of D-mannose (Fig. 9D, G). On the other hand, D-mannose itself did not affect the expression of OT and OTR mRNA in the cultured enteric neurons (Additional file 4: Fig. S4H). These data further prove our hypothesis that M2 macrophages promote the expression of the OT signalling system in the colon.

\section{Peg3 is involved in the upregulation of OT and OTR expression induced by $\mathrm{M} 2$ macrophage polarization} Peg3 (Paternally Expressed Gene 3) encodes a DNAbinding protein that acts as an inhibitory transcriptional regulator of the expression of OTR [39]. Therefore, we hypothesized that cytokines released from polarized macrophages might influence OT signalling system 


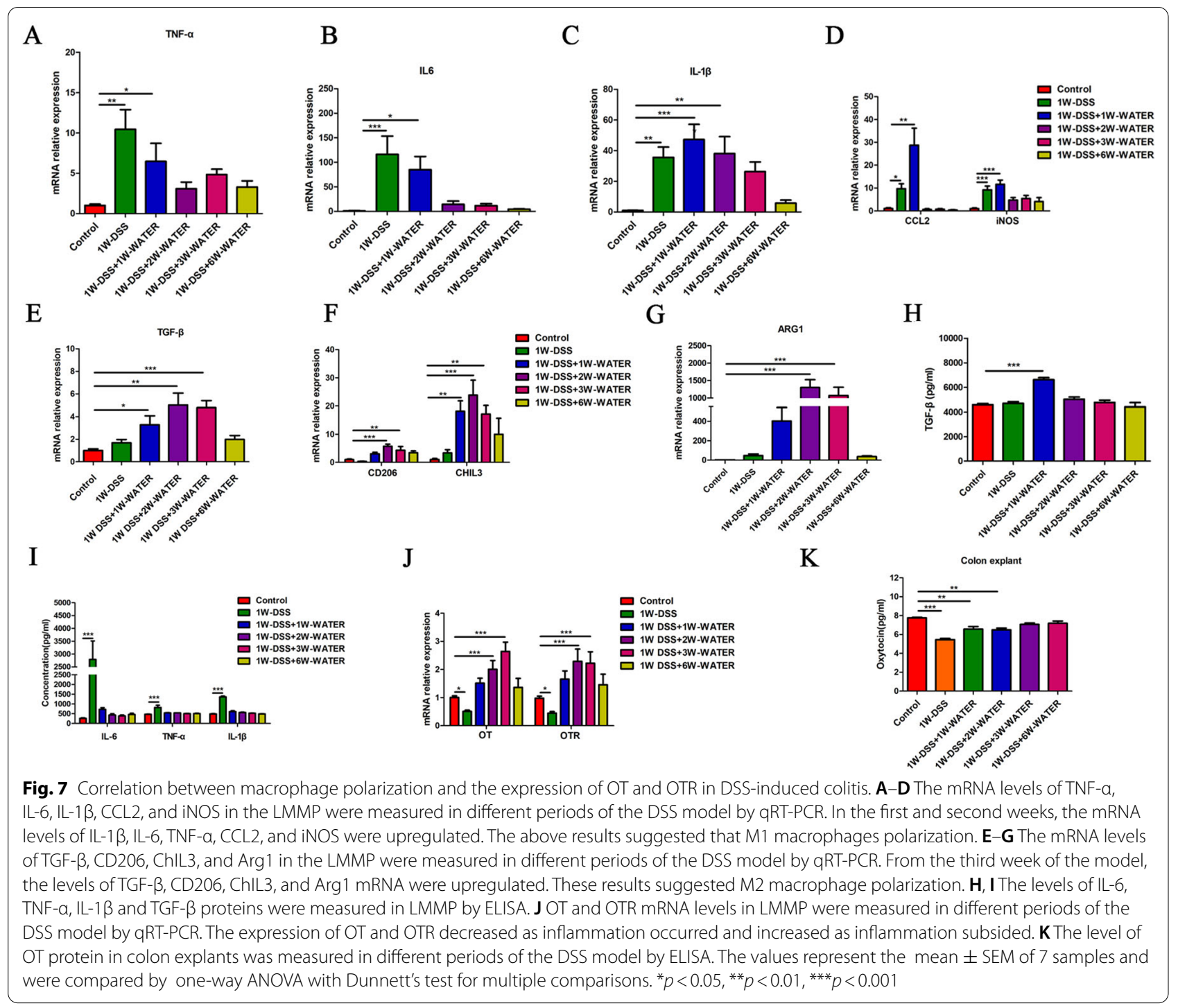

expression through Peg3. To prove this hypothesis, we first tested the expression of Peg3 and OT in D-mannosetreated mice. Seven days following D-mannose treatment, the expression of Peg3 was inhibited, whereas the expression of OT was increased (Additional file 4: Fig. S4K and Fig. 10A, B). Because TGF- $\beta$ promotes the expression of the OT signalling system through the Smad2/3 signalling pathway, we investigated whether the downregulation of Peg3 expression following D-mannose treatment also occurred through Smad2/3. We found that injection of SIS3CHL, a Smad3 blocker, significantly abolished the effect of D-mannose on the expression of Peg3 (Fig. 10C-E). We repeated this experiment in cultured enteric neurons and found that treatment with M0 and M1 supernatant and inflammatory cytokines, including IL- 6 , IL- $1 \beta$ and TNF- $\alpha$, did not change the expression level of Peg3. By contrast, treatment with M2 supernatant and TGF- $\beta$ significantly reduced Peg3 expression compared with the control group of cultured enteric neurons (Fig. 10F, G). Blocking the Smad3 signalling pathway with SIS3HCI significantly attenuated the downregulation of Peg3 expression and upregulation of OT and OTR expression (Fig. 10H, I). These results indicate that M2 macrophages and TGF- $\beta$ increase OT and OTR expression through downregulation of Peg3 expression.

\section{Discussion}

Our current study demonstrates that macrophage polarization affects the expression of the OT signalling system in the gut. In vitro, M1 macrophages inhibit the expression of the OT signalling system through secretion of proinflammatory factors, including IL- $1 \beta$, IL- 6 and TNF$\alpha$, and the intracellular STAT3 and NF- $\mathrm{kB}$ pathways in enteric neurons. On the other hand, M2 macrophages 


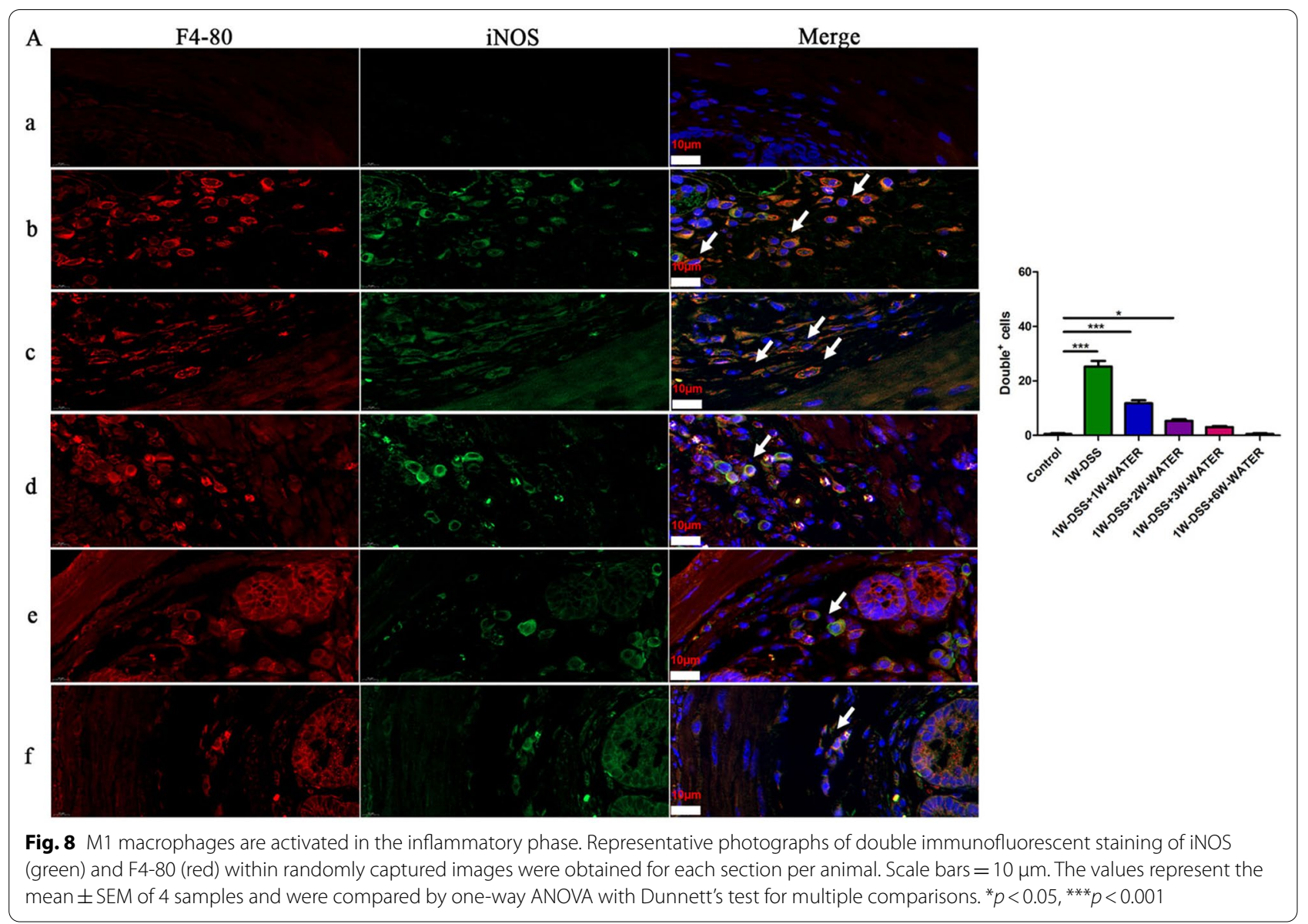

promote the expression of the OT signalling system in enteric neurons through secretion of TGF- $\beta$ and the intracellular Smad2/3-Peg3 pathway. This report is the first to demonstrate that macrophage polarization differentially regulates OT and OTR expression in enteric neurons (Fig. 11).

Levels of the stress hormone OT change during infection and inflammation [40-42]. In response to LPS stimulation, recruited M1 macrophages produce large amounts of proinflammatory cytokines, such as IL-1 $\beta$, IL- 6 , and TNF- $\alpha$. IL-4 treatment induces M2 macrophage polarization and the release of anti-inflammatory cytokines such as TGF- $\beta$ and IL-10 [43]. We found that proinflammatory cytokines, including IL- $1 \beta$, IL- 6 and TNF- $\alpha$, inhibited OT and OTR expression in enteric neurons, whereas TGF- $\beta$ had an excitatory effect. These data consistent with the observation that M1 supernatant inhibits OT and OTR expression and that M2 supernatant increases OT and OTR expression. This result was also verified in enteric neurons extracted from female mice. We therefore concluded that the cytokines in the M1 supernatant that inhibit OT and OTR expression in enteric neurons might be IL-1 $\beta$, IL- 6 and TNF- $\alpha$, while the cytokines in
M2 supernatant that increase OT and OTR expression might be TGF- $\beta$. Pretreatment with receptor antagonists of these cytokines abolished the effect of M1 and M2 supernatant. Therefore, we believe that following activation, M1 macrophages decrease OT and OTR expression in enteric neurons through the proinflammatory cytokines IL- $1 \beta$, IL- 6 and TNF- $\alpha$, while M2 macrophages increase OT and OTR expression through the anti-inflammatory cytokine TGF- $\beta$. After these inflammatory factors bind to their receptors, they participate in biological processes through a series of signalling pathways, such as JAK-STAT3 and NF- $\mathrm{KB}[30,31,44,45]$. In agreement with the above observations, we observed that the proinflammatory factors IL- 6 and TNF- $\alpha$ activate the STAT3 signalling pathway and that IL- $1 \beta$ activates the NF- $\mathrm{KB}$ signalling pathway to inhibit the expression of OT and OTR. Data from experiments in which the STAT3/ NF- $\mathrm{KB}$ signalling pathway was blocked further support these results. One of the interesting findings of the present study is that M2-polarized macrophages increase the expression of OT and OTR in enteric neurons, which might be mediated by TGF- $\beta$. TGF- $\beta$ mainly regulates downstream cell responses through Smad and non-Smad 


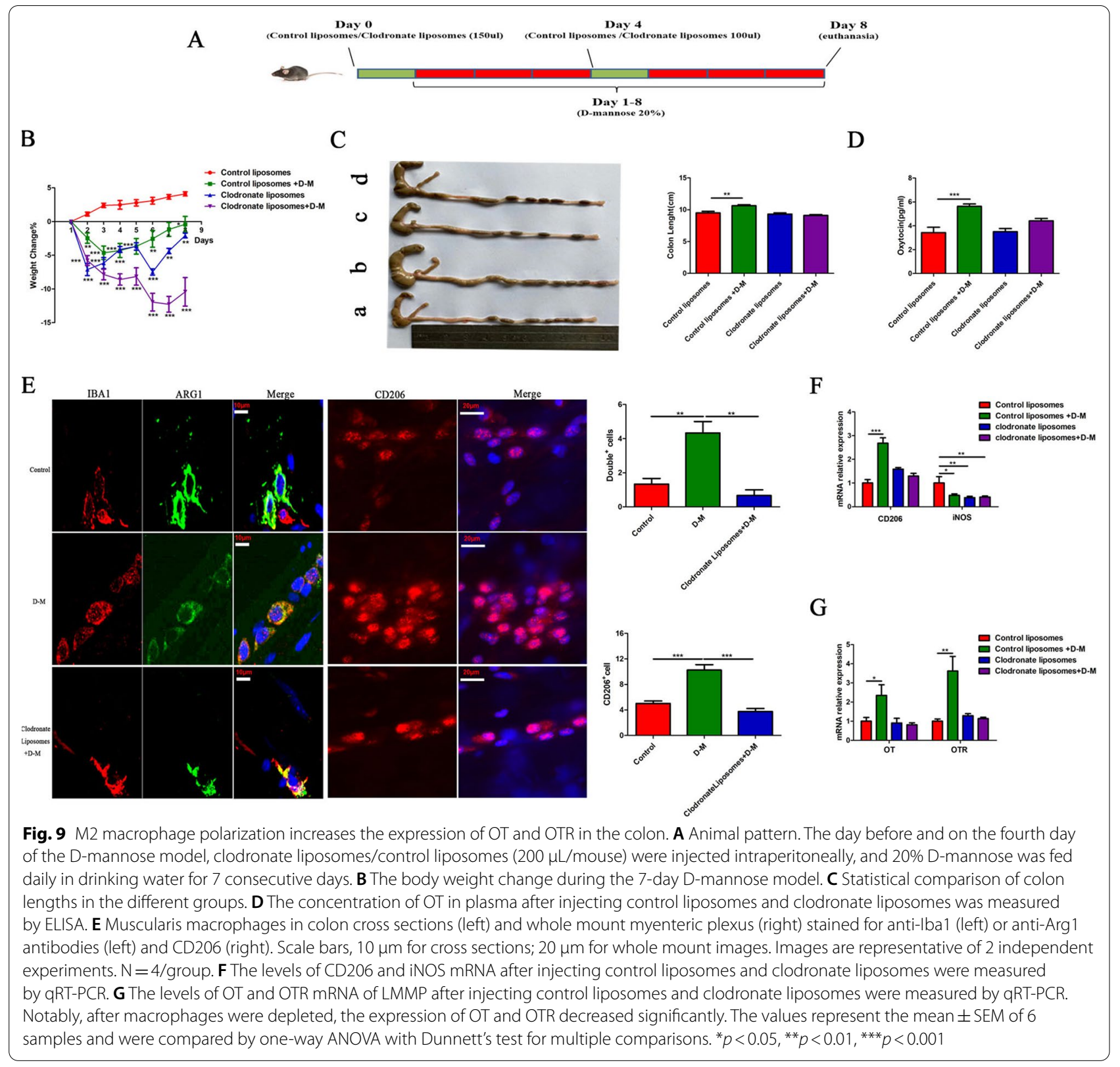

signalling pathways $[33,46]$. Our experiments confirmed that TGF- $\beta$ activates Smad2/3 and promotes the expression of OT and OTR. Both LY2109761 (TGF- $\beta$ receptor antagonist) and SIS3HCL (Smad3 blocker) completely abolished the excitatory effect of TGF- $\beta$ on OT and OTR. Therefore, we believe that TGF- $\beta$ upregulates the expression of OT and OTR in enteric neurons mainly through the intracellular Smad2/3 pathway.

To confirm the correlation between macrophage polarization and OT/OTR expression in vivo, we used a DSS-induced colitis model and investigated the temporal relationship between inflammation and OT/OTR expression. The disease activity index and the levels of proinflammatory cytokines in the colon increased and reached the highest level at the second week, so we defined this period as the inflammatory phase. In this period, the activation of M1 macrophages and the increase in proinflammatory cytokines were correlated with decreased OT and OTR expression in the ENS. Three weeks after DSS administration, the disease activity of the mice gradually returned to normal, and the expression of M2 macrophages markers and anti-inflammatory cytokines began to increase, reaching their highest level at the fourth week and returning to normal at 

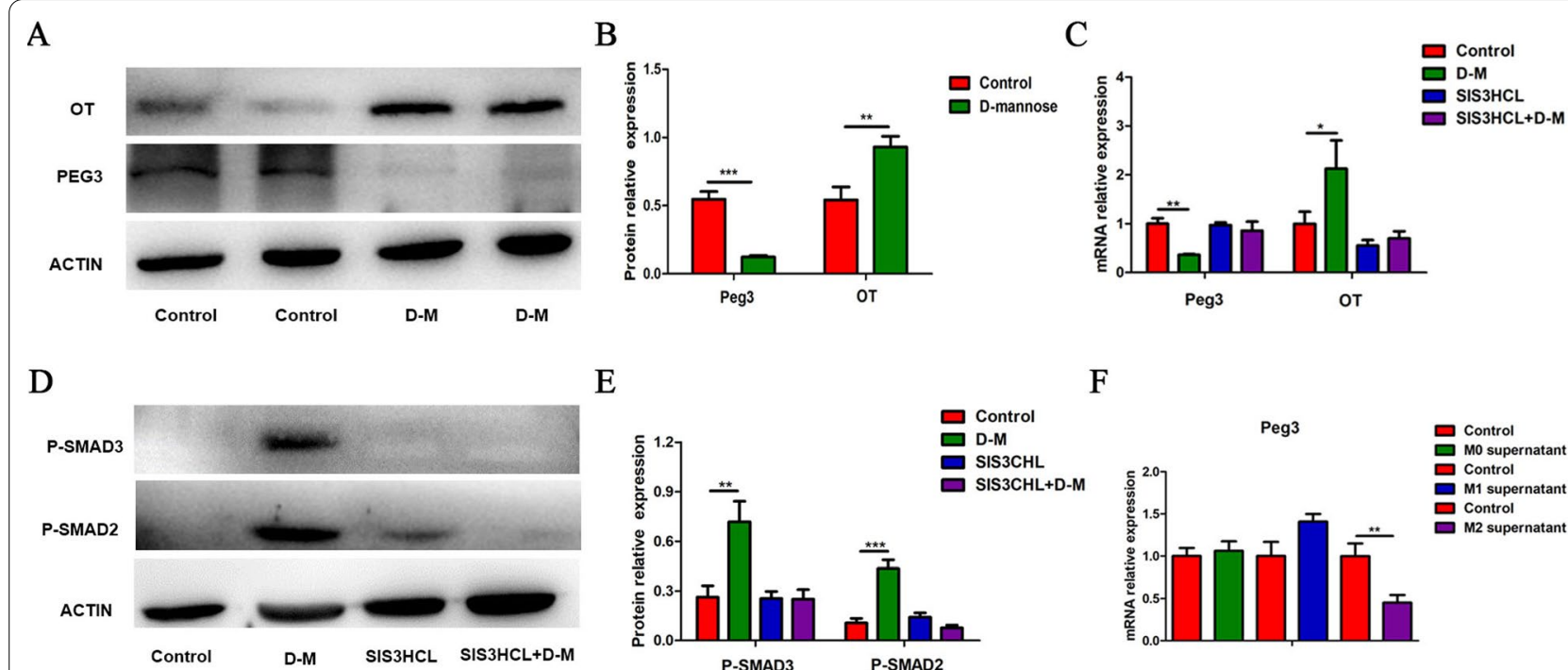

$\mathrm{E}$

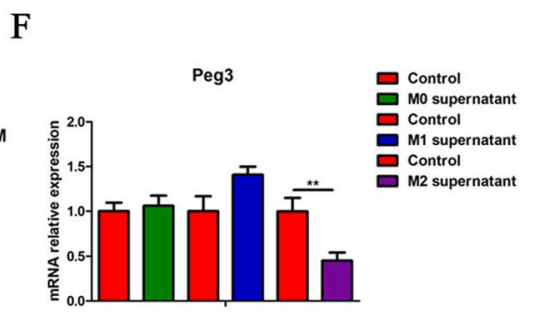

$\mathrm{H}$

I

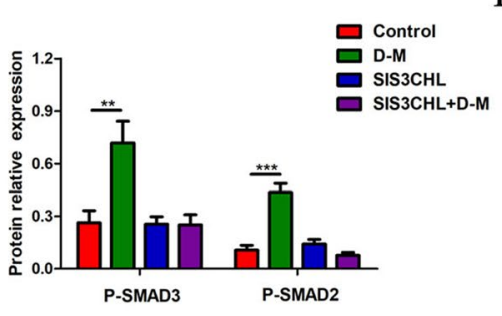

G
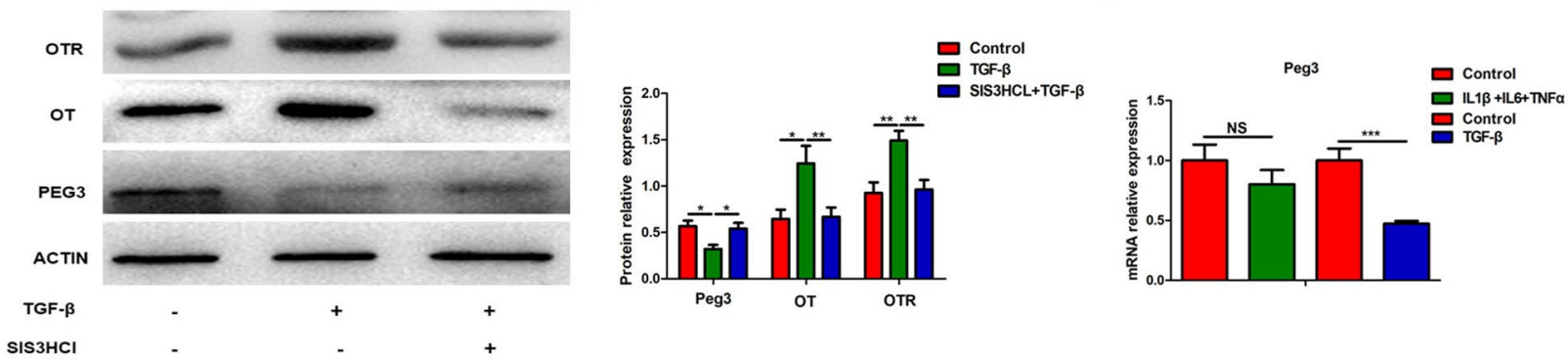

Fig. 10 Peg 3 is involved in the upregulation of OT and OTR expression induced by M2 macrophage polarization. A, B The expression of Peg3 and OT of LMMP in the D-mannose model was detected by western blot analysis. C The levels of Peg3 and OT mRNA of LMMP in the D-mannose model after injection of SIS3HCL (Smad3 inhibitor, $2.5 \mu \mathrm{g} / \mathrm{g}$ ) were measured by qRT-PCR. D, E Representative immunoblots for the detection of phosphorylated Smad2/3 after SIS3HCL injection by western blot analysis. $\beta$-actin was used to evaluate protein loading. F The level of Peg3 mRNA in cultured enteric neurons treated with or without conditioned medium was measured by qRT-PCR. M2 supernatant suppressed the expression of Peg3. G The level of Peg3 mRNA in cultured enteric neurons treated with or without IL-1 $\beta$ ( $1 \mathrm{ng} / \mathrm{ml})$, IL-6 (10 ng/ml), TNF-a (20 ng/ml) or TGF- $\beta$ $(10 \mathrm{ng} / \mathrm{ml})$ was measured by qRT-PCR. H, I Cultured enteric neurons were pretreated with SIS3CHL (Smad3 inhibitor, $5 \mu \mathrm{M}$ ) for $12 \mathrm{~h}$ followed by TGF- $\beta$ (10 ng/ml) for $24 \mathrm{~h}$. Only TGF- $\beta$ inhibited the expression of Peg3. IThe protein levels of Peg3, OT, and OTR were analysed by western blot, and $\beta$-actin was used to evaluate protein loading. The values represent the mean \pm SEM of 6 samples and were compared by $t$-test or one-way ANOVA with Dunnett's test for multiple comparisons. ${ }^{*} p<0.05,{ }^{* *} p<0.01,{ }^{* * *} p<0.001$

the fifth to seventh weeks. We defined this period as the recovery period. In this period, macrophage polarization to the M2 type and TGF- $\beta$ release were correlated with increased OT/OTR expression in the ENS. This observation further supports our hypothesis that following acute inflammation, dynamic changes in OT/OTR expression are correlated with differences in macrophage polarization in the gut. M1 macrophage polarization is correlated with decreased OT/OTR expression in the inflammatory period, whereas M2 macrophage polarization is correlated with increased OT/OTR expression in the recovery period. To the best of our knowledge, this study is the first to examine the dynamic changes in OT/OTR expression in the ENS following DSS-induced colitis. Although we found that OT inhibited the polarization of macrophages to the M1 type and promoted polarization to the M2 type and that OTR-deficient mice were more susceptible to DSS-induced colitis [22, 42], the anti-inflammatory effect of OT might not be very important during the acute inflammatory period. During this period, the release of proinflammatory cytokines from M1 macrophages was associated with decreased expression of OT and OTR in the ENS. However, interestingly, OT/ OTR expression increased during the recovery period, possibly due to polarization of M2 macrophages and the release of TGF- $\beta$. During the recovery period of acute inflammation, the polarization of M2 macrophages and the release of anti-inflammatory cytokines are primarily 


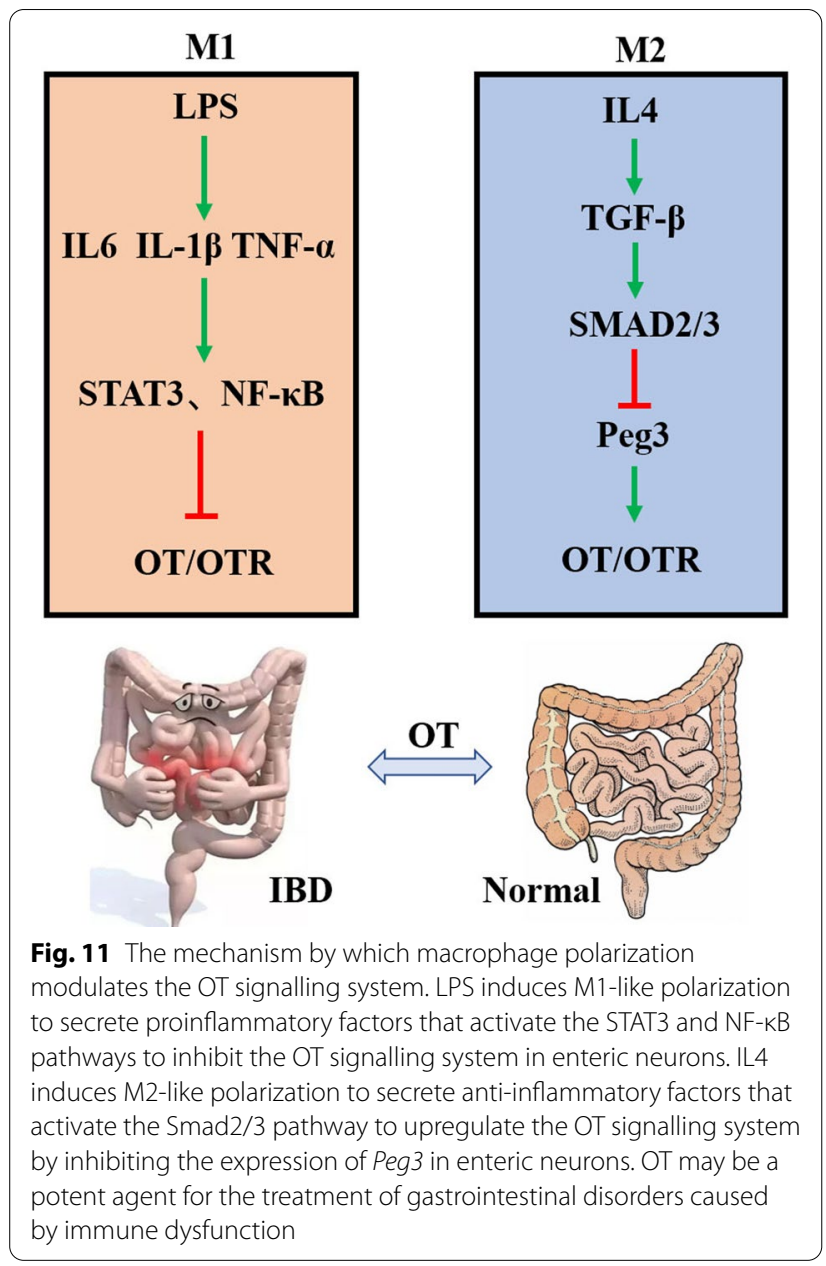

responsible for confining inflammation and promoting the proliferation of damaged tissue [14, 47-50]. During this period, there was positive feedback between M2 macrophage activity and the expression of OT and OTR in the ENS, so it is possible that the OT signalling system in the ENS is involved in tissue repair following local acute inflammation in the gut.

D-Mannose prevents acute lung injury by regulating PPAR- $\gamma$ and TGF- $\beta$ levels [25] and inhibits LPS-induced macrophage activation by impairing IL- $1 \beta$ production. In vivo, D-mannose improves endotoxaemia and relieves colitis [35]. Therefore, we used D-mannose to induce polarization of M2 macrophages in mice. Following D-mannose administration, the expression of iNOS in the gut was significantly downregulated, whereas TGF- $\beta$, CD206, and Arg1 expression were significantly increased. OT and OTR expression in the colon were also increased. The above data indicate that D-mannose inhibits the polarization of M1 macrophages and promotes the polarization of M2 macrophages. The increases in OT and OTR expression following treatment with D-mannose might be due to polarization of M2 macrophages. We also confirmed those results in female mice. To further test this hypothesis, we used clodronate liposomes to deplete macrophages in vivo. The upregulation of OT and OTR expression was abolished by the depletion of macrophages. Therefore, we believe that, in vivo, D-mannose increases OT and OTR expression in the ENS through polarization of macrophages to the M2 type. Another interesting finding in these experiments was that following D-mannose administration, the size and weight of the gut were increased, although the histological structure of the intestine did not change. These changes were macrophages dependent. Therefore, we concluded that these changes may have been caused by the release of cytokines such as TGF- $\beta$ from polarized M2 macrophages and OT released from the ENS.

Peg3 is widely expressed in various human and mouse tissues and encodes a DNA-binding protein that is involved in the control of specific target genes with different cell functions [51]. Peg3 also functions as a transcriptional regulator of the expression of OTR. In Peg3-KO mice, the expression of OTR in the hypothalamus and breast is upregulated [39]. In this study, we first proved that M2 supernatant and TGF- $\beta$ promoted the expression of OT by suppressing the expression of Peg3 in enteric neurons. However, M0/M1 macrophages and proinflammatory factors did not affect the expression of Peg3.

In recent years, studies have shown that OT has anti-inflammatory effects in addition to its effects on behaviour, lactation and childbirth [1,52]. OT acts as a neuropeptide to inhibit atherosclerosis and reduce inflammation of the visceral fat depot [53, 54]. The administration of exogenous oxytocin improves wound healing by reducing the concentration of cortisol [55]. Studies have also pointed out that OT improves the antioxidant status of colon tissue and improves colon damage [7]. We previously found that OT relieves colitis by promoting the polarization of anti-inflammatory macrophages [22]. Our results indicate that OT is likely an important signalling molecule between neurons and macrophages and may be a potential drug target for the treatment of intestinal diseases caused by immune abnormalities.

Many reports have indicated that the ENS cooperates with intestinal macrophages to maintain intestinal immune homeostasis [56, 57]. The unique colocalization of MMs and enteric neurons has attracted great attention from researchers [58]. Emerging data suggest that MMs secrete cytokines to protect nerves from damage and promote nerve regeneration [59]. The secretion of these cytokines might be the result of the interaction between the ENS and the immune system. In this study, 
we found that macrophage polarization affected the expression of the enteric neuron OT signalling system. In a recent study, we reported that the OT signalling system regulates macrophage polarization. The OT signalling system in the ENS is very likely to be the signalling molecule for the dialogue between intestinal macrophages and neurons. This "feedback loop" between macrophages and enteric neurons could be a pivotal "controller" that determines the tendency toward homeostasis or disease.

\section{Conclusion}

In summary, the present findings demonstrate that macrophage polarization affects the expression of OT signalling system in the gut. The OT signalling system may also be a potential drug target for the treatment of intestinal diseases caused by abnormal immune systems.

\begin{abstract}
Abbreviations
ENS: Enteric nervous system; LMMP: Longitudinal muscle myenteric plexus; OT: Oxytocin; OTR: Oxytocin receptor; DAPI: 4,6' Diamidino-2-phenylindole dihydrochloride hydrate; FBS: Foetal bovine serum; IHC: Immunohistochemistry; LPS: Lipopolysaccharide; qRT-PCR: Reverse transcriptase quantitative real-time PCR; MMs: Muscularis macrophages; IL: Interleukin; TNF-a: Tumour necrosis factor-a; TGF- $\beta$ : Transforming growth factor $\beta$; Arg1: Arginase 1; STAT3: Signal transducer and activator of transcription3; Chil3: Chitinase-like 3; DSS: Dextran sulfate sodium; D-M: D-mannose; IBD: Inflammatory bowel disease; iNOS: Inducible nitric oxide synthase.
\end{abstract}

\section{Supplementary Information}

The online version contains supplementary material available at https://doi. org/10.1186/s12974-021-02313-w.

Additional file 1. Fig. S1. Effects of different drugs on the expression of OT signalling system in enteric neurons and IL $1 \beta$ does not affect the STAT3 pathway. (A, B) LPS (100 ng/ml) or IL-4 (10 ng/ml) stimulated cultured enteric neurons for $24 \mathrm{~h}$ did not affect levels of OT and OTR mRNA. (C) The secretion of OT was detected in cultured enteric neurons by ELISA. $(\mathrm{N}=4)$. (D) The effect of TGF- $\beta$ receptor inhibitor (LY2109761) on OT was measured by western blot. (E) To exclude the influence of the menstrual cycle, we extracted enteric neurons from bilateral ovariectomized (OVX) female mice one week after surgery [1]. The level of OT mRNA in cultured enteric neurons in female mice was detected with or without conditioned medium treatment for $24 \mathrm{~h}$ via QRT-PCR. (F) The activation of IL-1 $\beta$ on STAT3 signalling pathway was detected by western blot. The values represent the mean \pm SEM of 6 samples and were compared by $t$-test or one-way ANOVA with Newman-Keuls for multiple comparisons. ${ }^{*} p<$ $0.05,{ }^{* * *} p<0.001$ vs. control group.

Additional file 2. Fig. S2. Dynamic changes of various parameters of DSS-induced enteritis model over time. (A)Schematic diagram of mouse colitis model (Inflammation and recovery periods). The mice drank 2.5\% DSS for 7 consecutive days, and drank water from the second week. (B) Body weight changed of WT mice in different periods of the DSS model. (C, D) The lengths of the colon from different groups were statistically compared. (E) Representative disease activity index was counted in each group based on body weight loss, stool consistence and hematochezia. (F) Representative H\&E staining colonic section in each group (Scale bar: $100 \mu \mathrm{m})$. (G) Histological assessment of the indicated group. The values represent the mean \pm SEM of 7 samples and were compared by $t$-test or one-way ANOVA with Dunnett's testfor multiple comparisons. ${ }^{*} p<0.05$, *** $p<0.001$ vs. control group.
Additional file 3. Fig. S3. The changes of neuron density in each ganglion at different periods of the DSS model. Representative photographs of immunofluorescent staining of tubulin (green) within randomly captured images were obtained for each section per animal. (Scale bar: $10 \mu \mathrm{m})$. The values represent the mean \pm SEM of 4 samples and were compared by one-way ANOVA with Dunnett's test for multiple comparisons. ${ }^{* *} p<0.01$ vs. control group.

Additional file 4. Fig. S4. D-Mannose promoted polarization of M2 macrophages and inhibited the polarization of M1 macrophages. (A, B) Changes in weight and eating habits in D-mannose model. (C) Representative H\&E staining colonic section in each group (Scale bar: $100 \mu \mathrm{m})$. (D) The lengths of the colon from different groups were statistically compared. (E) The levels of CD206, Arg1, TGF- $\beta$, IL10, Chil3, YM1 mRNA of LMMP induced by D-mannose for 7 days were measured by qRT-PCR. (F) The levels of iNOS, IL-1 $\beta$, IL-6, TNF-a mRNA of LMMP induced by D-mannose for 7 days were measured by qRT-PCR. (G) The levels of OT and OTR mRNA of LMMP induced by D-mannose for 7 days were measured by qRT-PCR. $(\mathrm{H})$ The levels of OT and OTR mRNA by D-mannose for $24 \mathrm{~h}$ in enteric neurons were measured by qRT-PCR. (I) Eating habits of mice had no change during the 7 days D-mannose model. $(J)$ The level of Peg3 mRNA of LMMP in D-mannose model was measured by qRT-PCR. The expression of Peg3 of LMMP in the D-mannose group was significantly down-regulated compared with the control group (K) The level of Peg3 mRNA of LMMP in D-mannose model was measured by qRT-PCR. The expression of Peg3 of LMMP in the D-mannose group was significantly down-regulated compared with the control group. The values represent the mean \pm SEM of 6 samples and were compared by $t$-test for multiple comparisons. ${ }^{*} p<0.05,{ }^{* *} p<0.01,{ }^{* * *} p<0.001$ vs. control group.

Additional file 5. Fig. S5. The expression of OT was significantly increased in OVX-female mice after drinking D-mannose for 7 days. (A) The change of colon length in female mice. (B) The level of OT mRNA was detected by QPCR in OVX-female mice after drinking D-mannose for 7 days. The values represent the mean \pm SEM of 6 samples and were compared by $t$-test for multiple comparisons. ${ }^{* *} p<0.01,{ }^{* * *} p<0.001$.

\section{Acknowledgements}

We thank the animal medicine center of Shandong University for providing experimental animals and the Basic Medical Sciences of Shandong University for providing experimental places and basic materials.

\section{Authors' contributions}

CYL: made substantial contributions to study design, data interpretation, writing and revising of the manuscript, and final revision of the manuscript; YS: made substantial contributions to the laboratory work, analyses of data and editing of the manuscript; SL and HJZ: performed animal model; JCZ and TTC performed cells culture; BY and JXL: manuscript proof reading and editing. All authors read and approved the final manuscript.

\section{Funding}

This work was supported by grants from the National Scientific Foundation of China [Grant numbers NSFC31871160 and 32071127].

\section{Availability of data and materials}

The data generated during our study cannot be made publicly available due to the data safety concern. But the data are available from the corresponding author on reasonable request.

\section{Declarations}

\section{Ethics approval and consent to participate}

We conducted animal experiments according to International Guiding. Principle for Animal Research that were stipulated by the Council for International Organizations of Medical Sciences (CIOMS), the routines have obtained approval from Shandong University Animal Ethics Committee. The participants working with the animal model received training abiding by the rules of Institutional Animal Care and Use Committee Guidebook (IACUC). And written informed consent was obtained from all participants. 


\section{Consent for publication}

Not applicable. This article does not contain any individual person's data.

\section{Competing interests}

The authors declare that they have no competing interests.

\begin{abstract}
Author details
'Department of Physiology, School of Basic Medical Sciences, Cheeloo Medical College, Shandong University, 44 Wenhua Xi Road, Jinan 250012, Shandong, People's Republic of China. ${ }^{2}$ School of Biological Science and Technology, Jinan University, 336 Nanxinzhuang Xi Road, Jinan 250012, People's Republic of China. ${ }^{3}$ Jinan Central Hospital Affiliated to Shandong University, Jinan 250012, Shandong, People's Republic of China. ${ }^{4}$ Provincial Key Lab of Mental Disorders, Shandong University, Jinan 250012, Shandong, People's Republic of China.
\end{abstract}

Received: 9 June 2021 Accepted: 29 October 2021

Published online: 08 November 2021

\section{References}

1. Sala NL, et al. Oxytocin reproducing reflex milk ejection in lactating women. J Appl Physiol. 1974;36(2):154-8

2. Gimpl G, Fahrenholz F. The oxytocin receptor system: structure, function, and regulation. Physiol Rev. 2001;81(2):629-83.

3. Jurek B, Neumann ID. The oxytocin receptor: from intracellular signaling to behavior. Physiol Rev. 2018:98(3):1805-908.

4. Yang HP, et al. Nonsocial functions of hypothalamic oxytocin. ISRN Neurosci. 2013;2013: 179272.

5. LiT, et al. Approaches mediating oxytocin regulation of the immune system. Front Immunol. 2016;7:693.

6. Jin $\mathrm{D}$, et al. CD38 is critical for social behaviour by regulating oxytocin secretion. Nature. 2007;446(7131):41-5.

7. Iseri SO, et al. Oxytocin ameliorates oxidative colonic inflammation by a neutrophil-dependent mechanism. Peptides. 2005;26(3):483-91.

8. Jafarzadeh $\mathrm{N}$, et al. Oxytocin improves proliferation and neural differentiation of adipose tissue-derived stem cells. Neurosci Lett. 2014;564:105-10.

9. Che T, et al. Oxytocin hyperpolarizes cultured duodenum myenteric intrinsic primary afferent neurons by opening BK(Ca) channels through IP(3) pathway. J Neurochem. 2012;121(4):516-25.

10. Chen $D$, et al. Oxytocin evokes a pulsatile PGE2 release from ileum mucosa and is required for repair of intestinal epithelium after injury. Sci Rep. 2015;5:11731.

11. Gong $L$, et al. The antinociception of oxytocin on colonic hypersensitivity in rats was mediated by inhibition of mast cell degranulation via $\mathrm{Ca}(2+)$ NOS pathway. Sci Rep. 2016;6:31452.

12. Welch $M G$, et al. Expression and developmental regulation of oxytocin (OT) and oxytocin receptors (OTR) in the enteric nervous system (ENS) and intestinal epithelium. J Comp Neurol. 2009;512(2):256-70.

13. Mikkelsen HB. Macrophages in the external muscle layers of mammalian intestines. Histol Histopathol. 1995;10(3):719-36.

14. Zhu W, et al. Disequilibrium of M1 and M2 macrophages correlates with the development of experimental inflammatory bowel diseases. Immunol Invest. 2014;43(7):638-52.

15. Ai $L$, et al. Synbindin restrains proinflammatory macrophage activation against microbiota and mucosal inflammation during colitis. Gut. 2021. https://doi.org/10.1136/gutjnl-2020-321094.

16. Phillips RJ, Powley TL. Macrophages associated with the intrinsic and extrinsic autonomic innervation of the rat gastrointestinal tract. Auton Neurosci. 2012;169(1):12-27.

17. Matteoli $\mathrm{G}$, et al. A distinct vagal anti-inflammatory pathway modulates intestinal muscularis resident macrophages independent of the spleen. Gut. 2014;63(6):938-48.

18. Meroni $\mathrm{E}$, et al. Intestinal macrophages and their interaction with the enteric nervous system in health and inflammatory bowel disease. Acta Physiol (Oxf). 2019;225(3): e13163.

19. Muller PA, et al. Crosstalk between muscularis macrophages and enteric neurons regulates gastrointestinal motility. Cell. 2014;158(2):300-13.

20. Hoover DB. Cholinergic modulation of the immune system presents new approaches for treating inflammation. Pharmacol Ther. 2017;179:1-16.
21. Matheis F, et al. Adrenergic signaling in muscularis macrophages limits infection-induced neuronal loss. Cell. 2020;180(1):64-78 e16.

22. Tang $Y$, et al. Oxytocin system alleviates intestinal inflammation by regulating macrophages polarization in experimental colitis. Clin Sci (Lond). 2019:133(18):1977-92.

23. Lourenssen S, Miller KG, Blennerhassett MG. Discrete responses of myenteric neurons to structural and functional damage by neurotoxins in vitro. Am J Physiol Gastrointest Liver Physiol. 2009;297(1):G228-39.

24. Asano K, et al. Intestinal CD169(+) macrophages initiate mucosal inflammation by secreting CCL8 that recruits inflammatory monocytes. Nat Commun. 2015;6:7802.

25. Zhang D, et al. D-mannose induces regulatory T cells and suppresses immunopathology. Nat Med. 2017;23(9):1036-45.

26. Jokela TA, et al. Mannose reduces hyaluronan and leukocytes in wound granulation tissue and inhibits migration and hyaluronan-dependent monocyte binding. Wound Repair Regen. 2013;21(2):247-55.

27. Kumamaru $\mathrm{H}$, et al. Liposomal clodronate selectively eliminates microglia from primary astrocyte cultures. J Neuroinflammation. 2012;9:116.

28. Lalancette-Hebert M, et al. Selective ablation of proliferating microglial cells exacerbates ischemic injury in the brain. J Neurosci. 2007;27(10):2596-605.

29. Dora D, et al. Evidence of a myenteric plexus barrier and its macrophagedependent degradation during murine colitis: implications in enteric neuroinflammation. Cell Mol Gastroenterol Hepatol. 2021. https://doi. org/10.1016/j.jcmgh.2021.07.003.

30. Li B, et al. Increased hepcidin in hemorrhagic plaques correlates with iron-stimulated IL-6/STAT3 pathway activation in macrophages. Biochem Biophys Res Commun. 2019;515(2):394-400.

31. Atreya I, Atreya R, Neurath MF. NF-kappaB in inflammatory bowel disease J Intern Med. 2008;263(6):591-6.

32. Fransvea $\mathrm{E}$, et al. Blocking transforming growth factor-beta up-regulates E-cadherin and reduces migration and invasion of hepatocellular carcinoma cells. Hepatology. 2008;47(5):1557-66.

33. Miyake T, Aziz A, McDermott JC. Maintenance of the undifferentiated state in myogenic progenitor cells by TGFbeta signaling is smad independent and requires MEK activation. Int J Mol Sci. 2020. https://doi.org/ 10.3390/ijms21031057.

34. Li J, et al. Oxytocin down-regulates mesenteric afferent sensitivity via the enteric OTR/nNOS/NO/KATP pathway in rat. Neurogastroenterol Motil. 2015;27(1):51-62.

35. Torretta S, et al. D-mannose suppresses macrophage IL-1 beta production. Nat Commun. 2020;11(1):6343.

36. Kumamaru $\mathrm{H}$, et al. Liposomal clodronate selectively eliminates microglia from primary astrocyte cultures. J Neuroinflamm. 2012. https://doi.org/ 10.1186/1742-2094-9-116.

37. Ma Y, et al. Polyethylenimine nanofibrous adsorbent for highly effective removal of anionic dyes from aqueous solution. Sci China Mater. 2016;59(1):38-50.

38. Schaefer $\mathrm{N}$, et al. Resident macrophages are involved in intestinal transplantation-associated inflammation and motoric dysfunction of the graft muscularis. Am J Transplant. 2007;7(5):1062-70.

39. Frey WD, et al. Oxytocin receptor is regulated by Peg3. PLoS ONE. 2018;13(8): e0202476.

40. Friebe-Hoffmann $\mathrm{U}$, et al. The influence of interleukin-1 beta on oxytocin signalling in primary cells of human decidua. Regul Pept. 2007;142(3):78-85.

41. Matsunaga W, et al. LPS-induced Fos expression in oxytocin and vasopressin neurons of the rat hypothalamus. Brain Res. 2000;858(1):9-18.

42. Dou D, et al. Oxytocin signalling in dendritic cells regulates immune tolerance in the intestine and alleviates DSS-induced colitis. Clin Sci (Lond). 2021;135(4):597-611.

43. Gordon S, Martinez FO. Alternative activation of macrophages: mechanism and functions. Immunity. 2010;32(5):593-604.

44. Lawrence T, Natoli G. Transcriptional regulation of macrophage polarization: enabling diversity with identity. Nat Rev Immunol. 2011;11(11):750-61.

45. Taniguchi K, et al. YAP-IL-6ST autoregulatory loop activated on APC loss controls colonic tumorigenesis. Proc Natl Acad Sci U S A. 2017;114(7):1643-8.

46. Huang F, et al. Knockdown of TBRG4 suppresses proliferation, invasion and promotes apoptosis of osteosarcoma cells by downregulating 
TGF-beta1 expression and PI3K/AKT signaling pathway. Arch Biochem Biophys. 2020;686: 108351.

47. Moreira TG, et al. CLA-supplemented diet accelerates experimental colorectal cancer by inducing TGF-beta-producing macrophages and T cells. Mucosal Immunol. 2019;12(1):188-99.

48. Zhang $\mathrm{Y}$, et al. ECM1 is an essential factor for the determination of $\mathrm{M} 1$ macrophage polarization in IBD in response to LPS stimulation. Proc Natl Acad Sci U S A. 2020;117(6):3083-92.

49. Wang $X$, et al. The $\mathrm{m} 6 \mathrm{~A}$ reader IGF2BP2 regulates macrophage phenotypic activation and inflammatory diseases by stabilizing TSC1 and PPARgamma. Adv Sci (Weinh). 2021;8(13):2100209.

50. Asano K, et al. Intestinal CD169+ macrophages initiate mucosal inflammation by secreting CCL8 that recruits inflammatory monocytes. Nat Commun. 2015. https://doi.org/10.1038/ncomms8802.

51. Thiaville MM, et al. DNA-binding motif and target genes of the imprinted transcription factor PEG3. Gene. 2013;512(2):314-20.

52. Ferguson JN, et al. Oxytocin in the medial amygdala is essential for social recognition in the mouse. J Neurosci. 2001:21(20):8278-85.

53. Nation DA, et al. Oxytocin attenuates atherosclerosis and adipose tissue inflammation in socially isolated ApoE-/- mice. Psychosom Med. 2010;72(4):376-82.
54. Szeto A, et al. Oxytocin administration attenuates atherosclerosis and inflammation in Watanabe Heritable Hyperlipidemic rabbits. Psychoneuroendocrinology. 2013;38(5):685-93.

55. Detillion CE, et al. Social facilitation of wound healing. Psychoneuroendocrinology. 2004;29(8):1004-11.

56. Watkins LR, Maier SF. Immune regulation of central nervous system functions: from sickness responses to pathological pain. J Intern Med. 2005;257(2):139-55

57. Jarret $A$, et al. Enteric nervous system-derived IL-18 orchestrates mucosal barrier immunity. Cell. 2020;180(1):50-63 e12.

58. Gabanyi I, et al. Neuro-immune interactions drive tissue programming in intestinal macrophages. Cell. 2016;164(3):378-91.

59. Elabd C, et al. Oxytocin is an age-specific circulating hormone that is necessary for muscle maintenance and regeneration. Nat Commun. 2014;5:4082.

\section{Publisher's Note}

Springer Nature remains neutral with regard to jurisdictional claims in published maps and institutional affiliations.
Ready to submit your research? Choose BMC and benefit from:

- fast, convenient online submission

- thorough peer review by experienced researchers in your field

- rapid publication on acceptance

- support for research data, including large and complex data types

- gold Open Access which fosters wider collaboration and increased citations

- maximum visibility for your research: over $100 \mathrm{M}$ website views per year

At $\mathrm{BMC}$, research is always in progress.

Learn more biomedcentral.com/submissions 